POLIBETÁNICA

Núm. 39, pp. 175-215. México, 2015

\title{
PLANTAS ÚTILES DE LA COMUNIDAD INDÍGENA NUEVO SAN JUAN PARANGARICUTIRO, MICHOACÁN, MÉXICO
}

\section{USEFUL PLANTS OF INDIGENOUS COMMUNITY FROM NUEVO SAN JUAN PARANGARICUTIRO, MICHOACAN, MEXICO}

\author{
Miguel Ángel Bello-González ${ }^{1}$, Selene Hernández-Muñoz ${ }^{1}$, \\ Ma. Blanca Nieves Lara-Chávez ${ }^{1}$, y Rafael Salgado-Garciglia ${ }^{2}$ \\ ${ }^{1}$ Facultad de Agrobiología "Presidente Juárez" Universidad Michoacana de San Nicolás \\ de Hidalgo, Uruapan, Michoacán, México. \\ ${ }^{2}$ Instituto de Investigaciones Químico Biológicas, Universidad Michoacana de San Nicolás \\ de Hidalgo, Edif. B3, CP 58030, Morelia, Michoacán, México. \\ Correo electrónico: mabellog2@hotmail.com
}

\begin{abstract}
RESUMEN
La zona boscosa de Nuevo San Juan Parangaricutiro, Michoacán, México, se localiza entre los 1900 a los 3200 m.s.n.m. Se caracteriza por ser una región accidentada, con pendientes que varían del 5 al $80 \%$, predominan los suelos de tipo andosol húmico. El clima dominante es templado húmedo, con abundantes lluvias en verano. Los tipos de vegetación son el bosque de pino, bosque de pino-encino, bosque de pino-oyamel, bosque mesófilo de montaña. La heterogeneidad y la diversidad vegetal ha permitido a esta región de aproximadamente $183.18 \mathrm{~km}^{2}$, vincular la explotación del bosque con sus actividades socioeconómicas, utilizado las plantas del entorno para satisfacer sus necesidades. Teniendo en cuenta el conocimiento que tienen sobre sus recursos forestales y sus usos, es necesario sistematizar el conocimiento tradicional sobre la riqueza de recursos maderables y no maderables, a fin de optimizar su explotación y preservar tales recursos. El presente estudio tuvo como objetivo inventariar la
\end{abstract}

flora útil maderable y no maderable, para lo cual se realizaron 37 exploraciones de campo. Se elaboró un listado que incluye 59 familias 135 géneros y 246 especies, las familias más representativas fueron Asteraceae con 67 especies, Leguminosae con 23 especies y Gramineae con 20. De acuerdo al hábito de crecimiento vegetal, las herbáceas son las más utilizadas, seguidas por los arbustos y los árboles. Los usos registrados fueron medicinal, ceremonial, forrajero, maderable, ornamental, comestible, melífero, de uso doméstico, tóxico y de uso veterinario. Las partes de las plantas más utilizadas fueron la parte aérea, el tallo, la flor y la hoja. De las diferentes formas de empleo, la infusión, la planta aplicada directamente en la zona afecta, el macerado y la cataplasma fueron las más importantes de donde se extraen productos medicinales suministrados vía oral, cutánea y local; el aserrío y la planta guisada o consumida en fresco son otras formas de empleo. La principal forma de obtención de las plantas útiles fue la colecta. 
Palabras clave: plantas útiles no maderables, plantas útiles maderables, Nuevo San Juan Parangaricutiro.

\begin{abstract}
The study was carried out in the forest area of Nuevo San Juan Parangaricutiro (19 $21^{\circ}$ ' $00^{\prime \prime}$ and $19^{\circ} 34^{\prime} 45^{\prime \prime}$ North, longitude $102^{\circ}$ $08^{\prime} 15^{\prime \prime}$ and $102^{\circ} 17^{\prime} 30^{\prime \prime}$ ' West), Michoacan Mexico, with an approximate surface of $183.18 \mathrm{~km}^{2}$. The forests of the community are in altitudes from 1900 to $3200 \mathrm{~m}$. It is characterized to be an uneven region, with slopes that vary of 5 up to $80 \%$, the soil andosol humic prevail. The dominant climate is the $\mathrm{C}(\mathrm{m})(\mathrm{w}) \mathrm{big}$, temperate humid, with abundant rains in summer. The existent types of vegetation in the study area are: pine forest, forest of pine-oaks, forest of pine-sacred fir and montane rain forest. The present study had as objective to carry out an inventory of the flora useful timber and non timber, for 37 field explorations were carried out in periodic form during year and half, a total of 1050 specimens collected and taxonomically determined. A list was elaborated that includes 246 species useful timbers trees an non timber shrubs, 135 genus y 58 families, being the most representative the Asteraceae with 67 species, the Leguminosae with 23 species and Gramineae with 20 species. Inside the genus that contributed the biggest number of species they meet Quercus, Senecio, Salvia, Gnaphalium and Eupatorium.The herbaceous ones were the biological forms that contributed the biggest quantity in useful material, continued by the shrubs and the trees. The registered uses were medicinal, ceremonial, forage, timber, ornament, eatable, melliferous, domestic use, toxic and of use veterinarian. The part of the used
\end{abstract}

plant was the complete plant (aerial part), continued by the stem, the flower and the leaf. Of the various forms of employment, infusion, applied directly to the plant area affects the maceration and the poultice, were the most important of which medicinal products supplied oral, dermal and local route are extracted, the sawmill and stewed plant or consumed fresh are other forms of employment. The main way of obtaining useful plants was collected.

Key words: plants useful non timbers, plants useful timbers, Nuevo San Juan Parangaricutiro.

\section{INTRODUCCIÓN}

Las localidades que integran la Comunidad Indígena de Nuevo San Juan Parangaricutiro, Michoacán viven vinculadas al bosque $\mathrm{y}$, por lo tanto, han utilizado las plantas del entorno para satisfacer sus necesidades. El conocimiento que tienen sobre ellas, particularmente de la flora no maderable, se ha transmitido a través de numerosas generaciones aunque sin ningún registro, por lo que se desconocen las propiedades y usos que tienen las diferentes especies de plantas de la región. Debido a la estructura económica y social que prevalece en México, las nuevas generaciones menosprecian o ignoran este legado cultural, por lo que a corto plazo se podría perder irremediablemente (Page, 1995).

Muchas plantas medicinales utilizadas cotidianamente son objeto de sobrecolecta, disminuyendo sus áreas de distribución y sus posibilidades potenciales de aprovechamiento tales como Satureja macrostema (Benth.) Briq. (nurite), Helianthemum glomeratum Lag. (cenicilla), Heterotheca 
Bello González, M.A. et al.: Plantas útiles de la comunidad indígena Nvo. Sn. Juan Parangaricutiro, Mich., Méx.

inuloides Cass. (árnica), Tagetes filifolia Lag. (anis), Gnaphalium inornatum DC. (gordolobo), Lepechinia caulescens (Ortega) Epling. (chía) y Eryngium carlinae F. Delaroche. (hierba del sapo), entre otras (Bello y Salgado, 2007). Esta colecta de plantas forma parte del complemento en la economía doméstica indígena al diversificar la producción agropecuaria (plantas medicinales, comestibles, aromáticas, de ornato, frutales, hortalizas; ganado menor y mayor, y especies semidomesticadas como el venado, la chachalaca y el jabalí), siendo, por lo tanto, importante en la conservación del germoplasma regional (Alarcón-Cháires, 2001).

La vegetación y, en particular, la flora maderable de esta comunidad indígena ha sido aprovechada intensamente a lo largo de los años, en este proceso de degradación y deterioro, el hombre interviene como causa $\mathrm{y}$ agente en forma directa e indirecta, de manera preponderante por las diferentes actividades de cultivo, incendios, tala, apertura de caminos, establecimiento de centros de población, tendidos de líneas de transmisión, además de plagas y enfermedades que se transmiten por diversas vías (Madrigal, 1992). Entre 1976 y 2000, Michoacán ha perdido más de 200000 hectáreas de bosques, con una tasa de desforestación de 0.47 $\%$ por año (Mas et al., 2004).

Esto ha ocasionado que algunas especies maderables estén reduciendo sus poblaciones de manera importante hasta el riesgo de desaparecer como son las diversas especies de Pinus (pino), Abies (oyamel), Quercus (encinos), Tilia (sirimo), Garrya (azulillo), Clethra (pácata), Alnus (aile), Arbutus (madroño) y Carpinus (palo barranco, moralillo) (NOM-059-SEMARNAT-2001).
Debido al conocimiento que tiene la comunidad sobre las diversas especies útiles maderables y no maderables, es importante revalorar la información con la finalidad de plasmarla en escritos y que sirvan como referencia para reforzar nuevas opciones de conservación y manejo.Tomando en consideración lo anterior se tienen como objetivos obtener un listado de las plantas útiles maderables y no maderables y analizar el conocimiento etnobotánico.

\section{Origen étnico y actividad económica}

San Juan Nuevo es un pueblo de origen Purépecha de 18834 habitantes ubicado en el estado de Michoacán México, en la meseta purépecha muy cerca del volcán Paricutín. Es la cabecera del municipio de Nuevo Parangaricutiro. El pueblo fue reconstruido, después de la destrucción total, tras la erupción del Paricutín en 1943, razón por la cual también se le conoce como "El Pueblo Que Se Negó a Morir", su nombre oficial es Nuevo San Juan Parangaricutiro (INEGI, 2010). La actividad económica de la comunidad indígena se basa principalmente en el cultivo de maíz, alfalfa, frijol, chile, durazno, manzana, pera y aguacate en tierras de temporal, la crianza de ganado vacuno y caballar, además de la extracción de resina y el aprovechamiento de maderas de coníferas y otra especies (Velázquez, 2003). La comunidad tiene un profundo arraigo religioso, esto se remonta al primer tercio del siglo XVI, entre 1530 y 1535 , época en que los misioneros franciscanos fundaron otros pueblos en los alrededores de Tancítaro. Es fray Juan de San Miguel, miembro de la Segunda Audiencia, encabezada por el ilustre don Vasco de Quiroga, que se atribuye la fundación de San Juan Parangaricutiro (García, 2013). Con esta 
influencia, la población en la actualidad festeja acontecimientos religiosos importantes como la danza de los Cúrpites, celebraciones de semana santa con quema de castillos y fuegos pirotécnicos, jueves de Corpus Cristi (demostración de oficios del pueblo y baile en explanada municipal), fiesta patronal en honor a San Juan Bautista, fiesta en honor al "Señor de los milagros" y día de muertos (García op. cit.).

\section{Descripción del área de estudio}

El estudio se llevó a cabo en la zona boscosa de la Comunidad Indígena de Nuevo San Juan Parangaricutiro, ubicada en la Meseta Purépecha, Michoacán, a $12 \mathrm{~km}$ al sur de la ciudad de Uruapan (19 $21^{\circ}$, 00 " y $19^{\circ} 34^{\prime} 45^{\prime}$ " latitud Norte y $102^{\circ} 08^{\prime}$ 15 " y $102^{\circ} 17$ ' 30" longitud Oeste). La comunidad presenta una superficie total de 18318 ha (fig. 1).

La cadena montañosa que atraviesa la comunidad forma parte del Eje Volcánico Transversal, dentro de la subprovincia Volcánica Tarasca, presentando una topografía accidentada, con pendientes que van del 5 al $80 \%$, constituido por lomeríos y laderas muy pronunciadas, generalmente con exposición sureste. Dentro de las principales elevaciones destacan el cerro Prieto (2300 m.s.n.m.) y el cerro del Pario (2 910 m.s.n.m.); la zona presenta una elevación promedio de 1900 m.s.n.m. (Dirección Técnica Forestal de la Comunidad Indígena de Nuevo San Juan Parangaricutiro, Michoacán, 1988). Los suelos se formaron a partir de cenizas volcánicas, producto de las erupciones volcánicas ocurridas en el Plioceno-Cuaternario. La mayoría de los suelos son profundos formados por una capa de arena volcánica. Los tipos de suelos más importantes son: andosol húmico de textura mediana, andosol ócrico de textura gruesa, regosol dístrico de textura gruesa y faeozem háplico de textura mediana (Instituto Nacional de Estadística, Geografía e Informática, 1985). Debido a las características hidrográficas y de suelo (arena, brechas y lava) existe una gran permeabilidad al agua. Los escurrimientos de estas corrientes de absorción afloran principalmente en tres subcuencas: río Tepalcatepec, río Itzícuaro y río Cupatitzio (Dirección Técnica Forestal de la Comunidad Indígena de Nuevo San Juan Parangaricutiro, Michoacán, 1988). El clima predominante en la zona es el C (m) (w) big templado húmedo, con abundantes lluvias en verano (García, 1988). La precipitación del mes más seco es menor de $49 \mathrm{~mm}$ y la lluvia invernal es menor de 5\% con respecto a la precipitación total anual. El verano es fresco y largo, con temperatura media anual del mes más caliente inferior a $22^{\circ} \mathrm{C}$. Isotermal con una oscilación menor de $5^{\circ} \mathrm{C}$ y con marcha de temperatura tipo Ganges; el mes más caliente se presenta antes de julio. La precipitación media anual es de 1500 a 2000 $\mathrm{mm}$, la temperatura media anual es de $18^{\circ} \mathrm{C}$, con frecuencia de heladas entre 20-40 días anuales (García, 1988; Instituto Nacional de Estadística, Geografía e Informática, 1985). Los principales tipos de vegetación representados en el área de estudio son: bosque de pino, bosque de pino-encino, bosque de pino-oyamel y bosque mesófilo de montaña. Algunos de los principales géneros y especies de árboles que se encuentran representados son: Pinus pseudostrobus Lindl., P. montezumae Lamb., P. leiophylla Schl. \& Cham., P. michoacana Martínez., P. douglasiana Martínez, Abies religiosa (HBK) Schltdl. \& Cham., Quercus rugosa Née., Q. obtusata Humb. \& Bonpl., Q. 


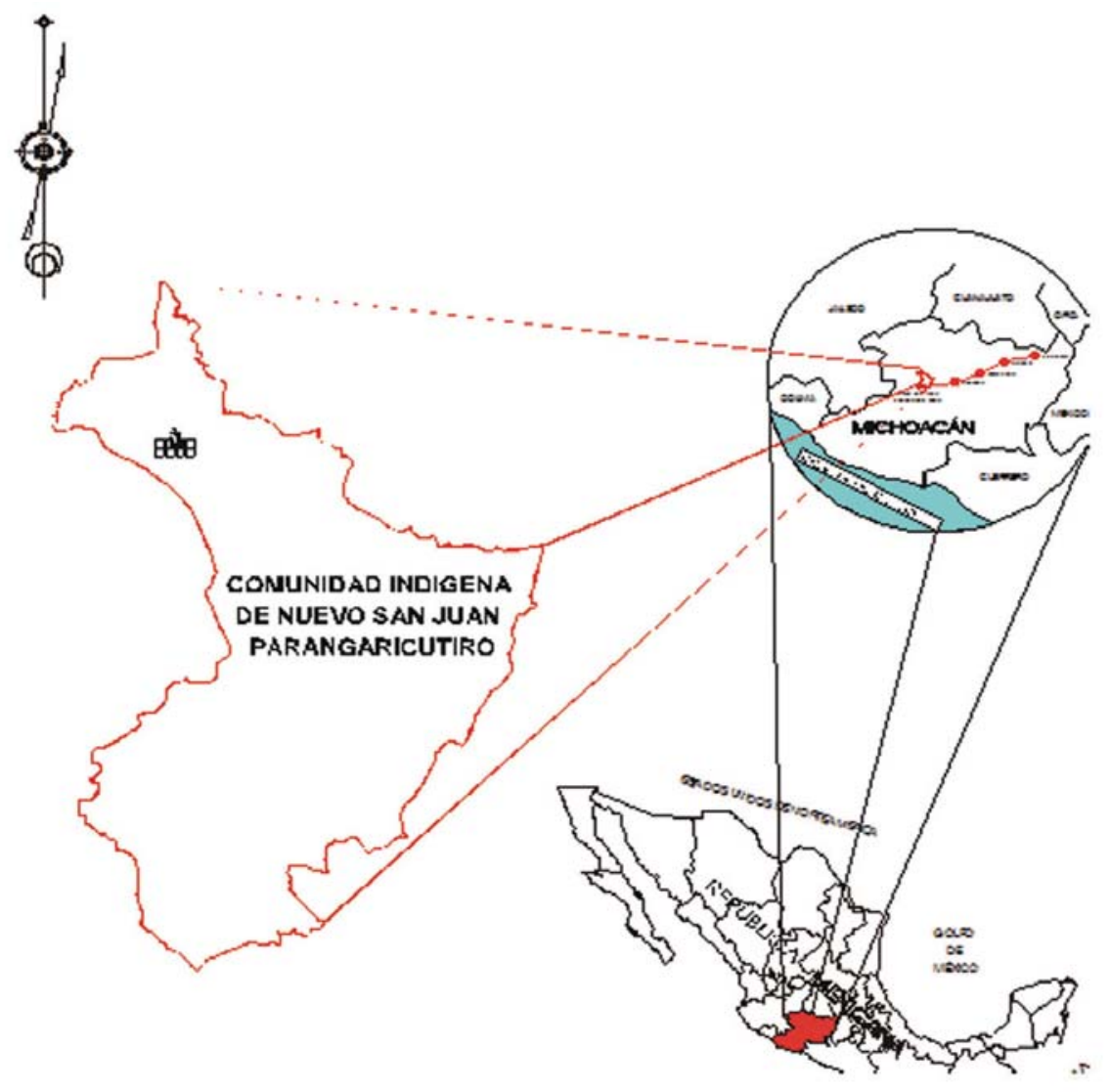

Fig. 1. Localización del área de estudio.

laurina Humb. \& Bonpl., Q. castanea Née., $Q$. candicans Née, $Q$. dysophylla Benth., Alnus jorullensis subsp. lutea Furlow., Carpinus caroliniana Walter., Tilia mexicana Schltdl y Ternstroemia pringlei (Rose) Standl. La vegetación de la zona ha sufrido cambios desde el punto de vista florístico, siendo en general heterogénea debido al aprovechamiento forestal del bosque. Los arbustos y hierbas se encuentran principal- mente en zonas deforestadas y en menor cantidad en bosques de pino-encino, llegando a desaparecer en la época de secas y a veces por completo. Los pastizales, por su parte, no constituyen un estrato uniforme, observándose algunos géneros como Andropogon, Muhlenbergia, Stipa, Lasciasis, Piptochaetium (Martínez, 1997; Medina, et al., 2000). La comunidad presenta una distribución aproximada de $77 \%$ de pino, 


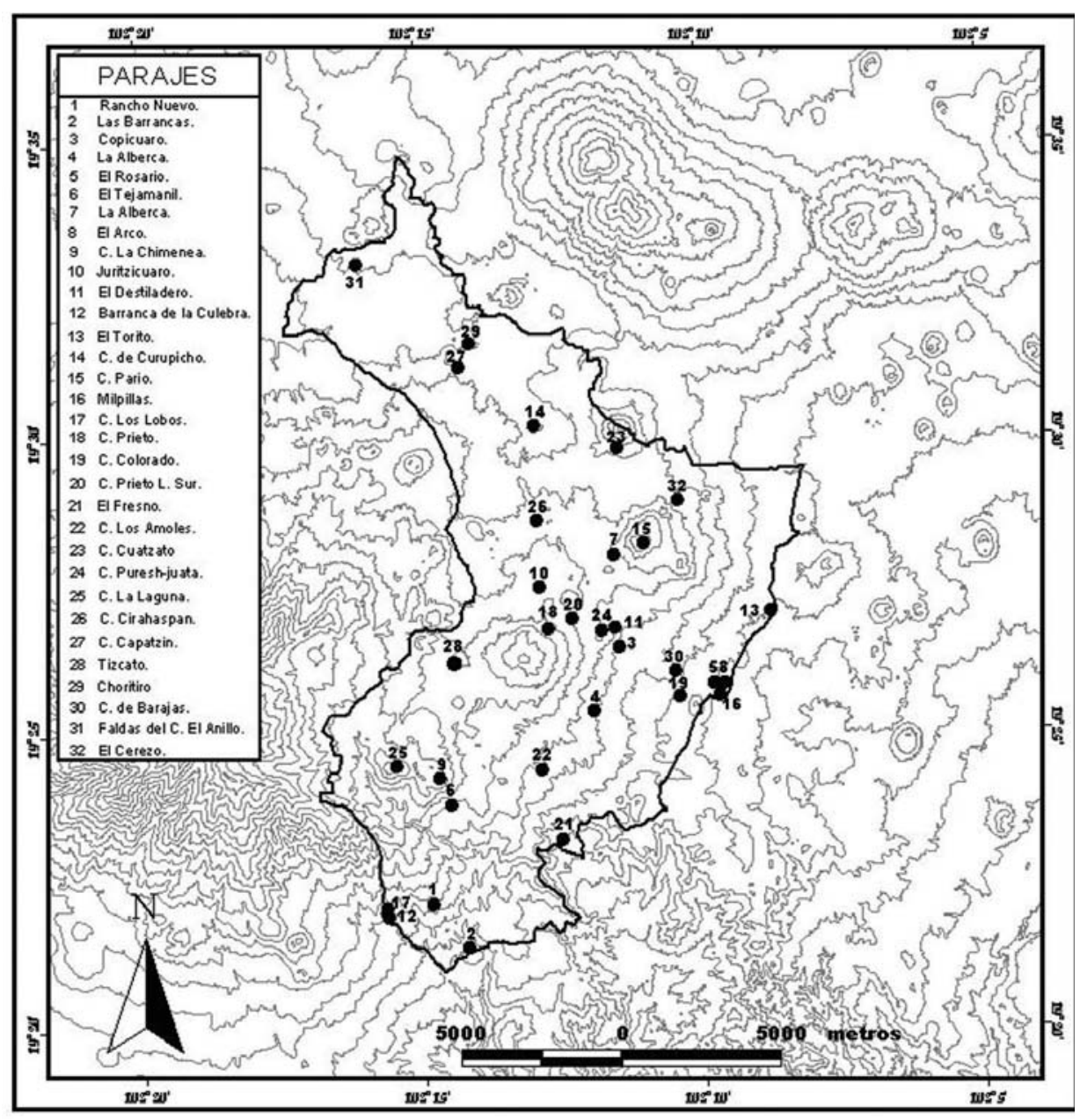

Fig. 2. Localidades exploradas en la Comunidad Indígena Nuevo San Juan Parangaricutiro, Michoacán. 
$12 \%$ de encino, $6 \%$ de oyamel y $5 \%$ de latifoliadas (Dirección Técnica Forestal de la Comunidad Indígena de Nuevo San Juan Parangaricutiro, Michoacán, 1988).

\section{MATERIAL Y MÉTODOS}

La metodología general del trabajo comprendió tres etapas:

\section{Etapa l}

Se revisó la información bibliográfica regional y se elaboró un formato para las colectas botánicas y las entrevistas, donde se consideraron los datos particulares de la planta y del medio ecológico.

\section{Etapa ll}

Se seleccionaron 32 sitios de colecta en localidades cercanas a algunos parajes dentro de la comunidad (fig. 2 y cuadro 1).

Posteriormente se realizaron las colectas botánicas y las entrevistas, éstas últimas son técnicas y herramientas para el acopio de información etnobotánica. Las entrevistas fueron aplicadas a 38 personas oriundas de la región y de prefencia de edad avanzada ( 25 personas mayores de 60 años), adultos (10 personal de 25 a 59 años de edad) y eventualmente se entrevistó a personas jóvenes (tres personas menores de 25 años). Se implementó la entrevista estructurada mediante la elaboración de cuestionarios, las cuales fueron usadas para el análisis cuantitativo, proporcionando respuestas verbales, las que pueden usarse sin necesidad de codificar o categorizar las respuestas utilizando para ello diversos métodos estadísticos (Cotton,1996). La entrevista contiene preguntas como nombre común de la planta en español y/o purépecha, parte utilizada, uso(s) de la planta, la forma de empleo. Para esta actividad se consideraron dos modalidades: a) se consultó a los informantes sobre el conocimiento de las plantas útiles y se procedió posteriormente al llenado de los formatos de registro y a la colecta; para ello se realizaron caminatas etnobotánicas, técnica que consiste en caminar con el informante a través de una o más zonas de vegetación colectando plantas y tomando notas. Debido a que las mismas plantas fueron mostradas a todos los informantes, los datos obtenidos mediante esta técnica pueden ser analizadas cuantitativamente (Martín, 2004), b) se realizó la colecta de todas las especies de plantas para posteriormente llevar a cabo la entrevista en el poblado más próximo.

\section{Etapa Ill}

Se procedió a determinar el material colectado mediante claves especializadas para los distintos grupos de plantas, así como su posterior cotejo con muestras botánicas depositadas en herbarios regionales. Se colectaron cinco ejemplares de cada espécimen con datos de campo: número de colecta, colector, lugar y características ecológicas o hábitat, estas muestras fueron depósitadas en el CE-Uruapan, Michoacán, INIFAP. Al final del artículo se incluye el apéndice correspondiente donde se agrupan las plantas útiles en orden jerárquico, además de los sitios (entre paréntesis) de distribución de las especies, referidos al cuadro 1, figura 2 y eventualmente algunas citas para complementar la información.

\section{RESULTADOS Y DISCUSIÓN}

Se registraron 59 familias, 135 géneros y 246 especies de plantas útiles maderables y no maderables, colectadas en los bosques templados de pino, pino-encino, 
Cuadro 1. Localidades exploradas en el área de la Comunidad Indígena Nuevo San Juan Parangaricutiro, Michoacán. México.

\begin{tabular}{|c|c|c|c|c|c|}
\hline Localidad & $\begin{array}{c}\text { Altitud } \\
(\text { m.s.n.m. })\end{array}$ & Vegetación & $\begin{array}{c}\text { Latitud } \\
\text { norte }\end{array}$ & $\begin{array}{c}\text { Longitud } \\
\text { oeste }\end{array}$ & Núm. \\
\hline Rancho Nuevo & 2250 & BPQ & $19^{\circ} 22^{\prime} 07.4^{\prime \prime}$ & $102^{\circ} 14^{\prime} 5^{\prime \prime}$ & 1 \\
\hline Las Barrancas & 2130 & BPQ & $19^{\circ} 21^{\prime} 21.9^{\prime \prime}$ & $102^{\circ} 14^{\prime} 13.6^{\prime \prime}$ & 2 \\
\hline Copicuaro & 2400 & BPQ & $19^{\circ} 26^{\prime} 26.7^{\prime \prime}$ & $102^{\circ} 11^{\prime} 28.3^{\prime \prime}$ & 3 \\
\hline La Alberca & 2500 & BPQ & $19^{\circ} 25^{\prime} 22.3^{\prime \prime}$ & $102^{\circ} 11^{\prime} 55.9^{\prime \prime}$ & 4 \\
\hline E1 Rosario & 2090 & BPQ & $19^{\circ} 25^{\prime} 48.3^{\prime \prime}$ & $102^{\circ} 09^{\prime} 47.1^{\prime \prime}$ & 5 \\
\hline El Tejamanil & 2480 & BPQ & $19^{\circ} 23^{\prime} 47.9^{\prime \prime}$ & $102^{\circ} 14^{\prime} 30^{\prime \prime}$ & 6 \\
\hline Rancho La Alberca & 2550 & BPQ & $19^{\circ} 27^{\prime} 59.1^{\prime \prime}$ & $102^{\circ} 11^{\prime} 32.8^{\prime \prime}$ & 7 \\
\hline El Arco & 2500 & $\mathrm{BP}$ & $19^{\circ} 25^{\prime} 22.3^{\prime \prime}$ & $102^{\circ} 08^{\prime} 11.3^{\prime \prime}$ & 8 \\
\hline Cerro La Chimenea & 2590 & $\mathrm{BA}$ & $19^{\circ} 24^{\prime} 15.2^{\prime \prime}$ & $102^{\circ} 14^{\prime} 42^{\prime \prime}$ & 9 \\
\hline Cerro Juritzicuaro & 2600 & BPQ & $19^{\circ} 27^{\prime} 28.3^{\prime \prime}$ & $102^{\circ} 12^{\prime} 52.6^{\prime \prime}$ & 10 \\
\hline El Destiladero & 2550 & BPQ & $19^{\circ} 26^{\prime} 45.7^{\prime \prime}$ & $102^{\circ} 1132.6^{\prime \prime}$ & 11 \\
\hline Barranca de la Culebra & 2220 & BMM & $19^{\circ} 21^{\prime} 51.2^{\prime \prime}$ & $102^{\circ} 15^{\prime} 41.6^{\prime \prime}$ & 12 \\
\hline E1 Torito & 2020 & BPQ & $19^{\circ} 27^{\prime} 01.1^{\prime \prime}$ & $102^{\circ} 08^{\prime} 46.4^{\prime \prime}$ & 13 \\
\hline Las Cocinas & 2315 & $\mathrm{BP}$ & $19^{\circ} 30^{\prime} 39.1^{\prime \prime}$ & $102^{\circ} 09^{\prime} 34.1{ }^{\prime \prime}$ & 14 \\
\hline Cerro Pario & 2790 & $\mathrm{BP}$ & $19^{\circ} 28^{\prime} 11.7^{\prime \prime}$ & $102^{\circ} 11^{\prime} 01.2^{\prime \prime}$ & 15 \\
\hline Milpillas & 2035 & BPQ & $19^{\circ} 24^{\prime} 53.1^{\prime \prime}$ & $102^{\circ} 09^{\prime} 03.8^{\prime \prime}$ & 16 \\
\hline Cerro Los Lobos & 2200 & $\mathrm{BP}$ & $19^{\circ} 21^{\prime} 51.3^{\prime \prime}$ & $102^{\circ} 15^{\prime} 41.7^{\prime \prime}$ & 17 \\
\hline Cerro Prieto (Este) & 2900 & $\mathrm{BP}$ & $19^{\circ} 26^{\prime} 46^{\prime \prime}$ & $102^{\circ} 12^{\prime} 44.7^{\prime \prime}$ & 18 \\
\hline Cerro Colorado & 2240 & BPQ & $19^{\circ} 25^{\prime} 34.7^{\prime \prime}$ & $102^{\circ} 10^{\prime} 24.0^{\prime \prime}$ & 19 \\
\hline Cerro Prieto (Oeste) & 2750 & $\mathrm{BP}$ & $19^{\circ} 26^{\prime} 55.1^{\prime \prime}$ & $102^{\circ} 12^{\prime} 18.9^{\prime \prime}$ & 20 \\
\hline E1 Fresno & 2200 & BPQ & $19^{\circ} 23^{\prime} 11.2^{\prime \prime}$ & $102^{\circ} 12^{\prime} 32.0^{\prime \prime}$ & 21 \\
\hline Cerro Los Amoles & 2500 & BPQ & $19^{\circ} 24^{\prime} 22.2^{\prime \prime}$ & $102^{\circ} 12^{\prime} 53.2^{\prime \prime}$ & 22 \\
\hline Cerro Cutzato & 2500 & BPQ & $19^{\circ} 29^{\prime} 48.1^{\prime \prime}$ & $102^{\circ} 11^{\prime} 27.3^{\prime \prime}$ & 23 \\
\hline Puresh-Juata & 2710 & BPQ & $19^{\circ} 26^{\prime} 43.5^{\prime \prime}$ & $102^{\circ} 11^{\prime} 47.5^{\prime \prime}$ & 24 \\
\hline Cerro La Laguna & 3040 & $\mathrm{BA}$ & $19^{\circ} 24^{\prime} 27.4^{\prime \prime}$ & $102^{\circ} 15^{\prime} 28.7^{\prime \prime}$ & 25 \\
\hline Cerro Cirahaspan & 2519 & BPQ & $19^{\circ} 31^{\prime} 11.5^{\prime \prime}$ & $102^{\circ} 14^{\prime} 16.9^{\prime \prime}$ & 26 \\
\hline Cerro Capatzin & 2366 & BPQ & $19^{\circ} 31^{\prime} 11.5^{\prime \prime}$ & $102^{\circ} 14^{\prime} 16.9^{\prime \prime}$ & 27 \\
\hline Cerro Tizcato & 2740 & BPQ & $19^{\circ} 26^{\prime} 12.4^{\prime \prime}$ & $102^{\circ} 14^{\prime} 24.3^{\prime \prime}$ & 28 \\
\hline Cerro Choritiro & 2310 & $\mathrm{BP}$ & $19^{\circ} 31^{\prime} 35.9^{\prime \prime}$ & $102^{\circ} 14^{\prime} 05.9^{\prime \prime}$ & 29 \\
\hline Cerro de Barajas & 2300 & $\mathrm{BP}$ & $19^{\circ} 26^{\prime} 00.3^{\prime \prime}$ & $102^{\circ} 14^{\prime} 05.9^{\prime \prime}$ & 30 \\
\hline Cerro del Anillo & 2260 & VS & $19^{\circ} 32^{\prime} 58.1^{\prime \prime}$ & $102^{\circ} 16^{\prime} 05.1^{\prime \prime}$ & 31 \\
\hline El Cerezo & 2430 & $\mathrm{BP}$ & $19^{\circ} 28^{\prime} 53.7^{\prime \prime}$ & $102^{\circ} 10^{\prime} 23.5^{\prime \prime}$ & 32 \\
\hline
\end{tabular}

$\mathrm{BP}=$ Bosque de pino, $\mathrm{BPQ}=$ Bosque de pino-encino, $\mathrm{BA}=$ Bosque de oyamel, $\mathrm{BMM}=$ Bosque mesófilo de montaña, $\mathrm{VS}=$ vegetación secundaria. 
oyamel, mesófilo de montaña y vegetación secundaria derivadas de los mismos. En el cuadro 2 se muestra el total de familias, géneros y especies de plantas útiles para la comunidad indígena Nuevo San Juan Parangaricutiro y su distribución dentro de los grandes grupos taxonómicos.

Dentro de esta distribución, las dicotiledóneas forman el grupo mejor representado, las familias, los géneros y las especies que la constituyen son comunes en otros lugares del país. Las familias de plantas con mayor número de especies útiles fueron Asteraceae, Leguminosae y Gramineae, juntas representan el $44.82 \%$ del total (cuadro 3). La presencia de estas familias en otras región del estado coinciden con Bello (1993), Díaz y Bello (1993), Bello y Madrigal (1996), Martínez (1997), Hernández (2005) y Rojas (2006).

El mayor uso de plantas dentro de las Asteraceae probablemente se deba a la abundancia y la diversidad de esta familia en México y en Michoacán (Rzedowski, 1991; Carranza, 2005). Los antecedentes regionales coinciden en citar a esta familia como la más abundante en los bosques templados de pino, encino, pino-encino, mesófilo de montaña, oyamel y vegetación secundaria derivada de éstos (Flores, 1987; Esparza, 1989; García, 1990; Bello, 1993; Díaz y Bello, 1993; Pérez, et al., 1994; Bello y Madrigal, 1996; Silva, 1996; Martínez, 1997; Suárez, 1997; Alvarado, 1999; Farfán, 2001; Hurtado y Rodríguez, 2001; Bernal, 2002; García, 2002; Delgado, 2003; Hernández, 2005; Rojas, 2006). En segundo y en tercer lugar de importancia por el número de especies que la constituyen, están las Leguminosas y las Gramíneas, distribuyéndose principalmente en la vegetación secundaria y en menor cantidad en el sotobosque de los bosques de pino $\mathrm{y}$ pino-encino. Los géneros con mayor número de especies en el área de estudio son: Quercus, Salvia, Gnaphalium y Senecio (cuadro 3). Inventarios florísticos y de plantas medicinales regionales muestran la dominancia de Quercus, Salvia y Gnaphalium (Torres, 1994; Martínez, 1997; Bello y Salgado, 2007) además de estar dentro de los 10 géneros más abundantes de las regiones montañosas de clima semihúmedo y fresco de México (Rzedowski, 1991) (cuadro 4).

Cuadro 2. Distribución taxonómica de las plantas útiles.

\begin{tabular}{|l|c|c|c|}
\hline \multicolumn{1}{|c|}{ División } & Familias & Géneros & Especies \\
\hline Pteridophyta & 4 & 4 & 7 \\
Gymnospermas & 2 & 3 & 6 \\
Angiospermas & & & \\
Monocotiledónea & 6 & 16 & 29 \\
Dicotiledóneas & 47 & 112 & 204 \\
\hline \multicolumn{1}{|c|}{ TOTAL } & $\mathbf{5 9}$ & $\mathbf{1 3 5}$ & $\mathbf{2 4 6}$ \\
\hline
\end{tabular}


Cuadro 3. Familias con mayor número de especies útiles.

\begin{tabular}{|l|c|c|c|}
\hline \multicolumn{1}{|c|}{ Familia } & Géneros & Especies & \% Especies \\
\hline Asteraceae & 27 & 67 & 27.35 \\
Leguminosae & 14 & 23 & 9.34 \\
Gramineae & 13 & 20 & 8.13 \\
Lamiaceae & 4 & 11 & 4.47 \\
Fagaceae & 1 & 9 & 3.65 \\
Solanaceae & 3 & 8 & 3.25 \\
Rosaceae & 5 & 7 & 2.84 \\
Polypodiaceae & 4 & 7 & 2.84 \\
Onagraceae & 4 & 7 & 2.84 \\
Pinaceae & 2 & 5 & 2.03 \\
\hline
\end{tabular}

Cuadro 4. Géneros con mayor número de especies útiles.

\begin{tabular}{|l|c|}
\hline \multicolumn{1}{|c|}{ Géneros } & Especies \\
\hline Quercus L. & 9 \\
Salvia L. & 8 \\
Gnaphalium L. & 8 \\
Senecio L. & 9 \\
Eupatorium L. & 7 \\
Bidens L. & 6 \\
Tagetes L. & 5 \\
Stevia Cav. & 5 \\
Pinus L. & 4 \\
Cirsium Mill. & 4 \\
Adiantum L. & 4 \\
Dalea Lucanus. & 4 \\
\hline
\end{tabular}

En el cuadro 5 se muestran las formas de crecimiento que presentaron las diferentes especies de plantas útiles, con predominancia de las herbáceas, muchas de ellas con hábitos ruderal y arvense. Los arbustos formaron el segundo estrato en importancia, grupo que contiene el mayor número de géneros endémicos de la flora fanero- gámica mexicana (Rzedowski, 1991). Los árboles se encuentran representados por 30 especies, con tan sólo cuatro especies menos de las reportadas en estudios florísticos regionales (Martínez, 1997; Medina, et al., 2000). Las plantas útiles epífitas, trepadoras y parásitas fueron escasas y poco utilizadas. 
Bello González, M.A. et al.: Plantas útiles de la comunidad indígena Nvo. Sn. Juan Parangaricutiro, Mich., Méx.

Cuadro 5. Formas de crecimiento de las especies de plantas útiles.

\begin{tabular}{|l|c|c|}
\hline $\begin{array}{c}\text { Formas de } \\
\text { crecimiento }\end{array}$ & Núm. de especies & \% \\
\hline hierbas & 165 & 67.07 \\
arbustos & 46 & 18.69 \\
árboles & 30 & 12.19 \\
trepadoras & 2 & 0.81 \\
parásitas & 2 & 0.81 \\
epífitas & 1 & 0.40 \\
\hline
\end{tabular}

En el cuadro 6 se puede apreciar un listado de 10 categorías de uso con el número de especies útiles registradas. Los resultados muestran la predominancia de las plantas medicinales, seguida por las plantas de uso ceremonial, forrajeras, maderables, ornamentales y comestibles, el resto de las categorías, melífera, tóxica, de usos doméstico y veterinario fueron poco utilizadas. El uso de plantas con fines ceremoniales es la segunda forma de utilización entre los pobladores de la Comunidad Nuevo San Juan Parangaricutiro, sus creencias y tradiciones religiosas forman parte importante de su vida. Ofrendan una diversidad de plantas para el adorno de altares y de coronas durante las festividades religiosas y del día de muertos. El número de plantas utilizadas para este propósito asciende a más de 30 especies, principalmente de la familia Asteraceae. La necesidad de forraje para el ganado es importante para los habitantes de esta región, constituye un recurso vital para la crianza de ganado bovino principalmente, animales que consumen gran cantidad de plantas silvestres mediante la práctica tradicional y ancestral del pastoreo cerril. El número de plantas forrajeras registradas es amplio principalmente especies de gramíneas. La madera ha sido un recurso importante para el desarrollo de la comunidad, pero también ha sido un factor de impacto en el deterioro del medio ambiente. Las especies con mayor demanda son principalmente especies de Pinus, Quercus y Abies, además de otras especies de los géneros Clethra, Alnus, Arbutus, Garrya, Buddleia, Styrax, Symplocos y Tilia, de donde se obtiene madera aserrada para la elaboración de muebles y artesanías. Los usos ornamental, comestible, melífero, doméstico, tóxico y de uso veterinario presentaron cifras inferiores al 5\%.

En el cuadro 7 se muestran los síntomas y enfermedades tratadas con plantas medicinales. El número de especies medicinales es amplio y se emplean principalmente en enfermedades del aparato digestivo (infecciones, dolor de estómago, gastritis, úlceras, frío en el estómago y afecciones de la boca); padecimientos de la piel (heridas, raspones, quemaduras, picadura o mordedura de animales ponzoñosos, llagas, granos infectados, espinillas, sarna o roña, caspa o seborrea); enfermedades 
Cuadro 6. Número de especies por categoría de uso.

\begin{tabular}{|l|c|c|}
\hline Categoría de uso & Núm. de especies & \% \\
\hline medicinal & 128 & 52.03 \\
ceremonial & 33 & 13.41 \\
forraje & 29 & 11.78 \\
maderable & 23 & 9.34 \\
ornamental & 11 & 4.47 \\
comestible & 11 & 4.47 \\
melífera & 4 & 1.62 \\
doméstico & 4 & 1.62 \\
tóxico & 2 & 0.81 \\
veterinario & 1 & 0.40 \\
\hline
\end{tabular}

Cuadro 7. Número de especies por síntomas y enfermedades.

\begin{tabular}{|l|c|c|}
\hline Síntomas y enfermedades & Especies & \% \\
\hline aparato digestivo & 47 & 36.71 \\
padecimientos de la piel & 27 & 21.09 \\
aparato respiratorio & 19 & 14.84 \\
aparato urinario & 11 & 8.59 \\
padecimiento generales & 8 & 6.25 \\
enfermedades del hígado & 6 & 4.68 \\
sistema circulatorio & 5 & 3.90 \\
sistema nervioso & 4 & 3.12 \\
padecimientos de la mujer & 1 & 0.78 \\
\hline
\end{tabular}

del aparato respiratorio (gripa, catarro, garganta irritada o inflamada, ronquera, anginas, bronquios, asma y pulmones); enfermedades del aparato urinario (dolor o ardor al orinar, inflamación del riñón y la vejiga, orina escasa, piedras e impurezas en el riñón); padecimientos generales (dolor y punzada en la cabeza, fiebre o calentura, dolor de oídos e inflamación de ojos e impurezas en la sangre); enfermedades del hígado (dolores, inflamación congestión del hígado y vesícula, piedras en la vesícula); enfermedades del sistema circulatorio (mala circulación, presión arterial, artritis, reumas, calambres y moretones); enfermedades del sistema nervioso (nervios alterados) y padecimientos de la mujer (cólicos menstruales, parto difícil).

Las enfermedades o afecciones que más sufría la población indígena en los tiempos prehispánicos fueron los padecimientos de 
la piel y enfermedades del aparato digestivo (Caballero y Mapes, 1985; Sepúlveda, 1996). Información reciente señala que las enfermedades digestivas como diarreas, infecciones estomacales, dolores de estómago, gastritis, pesadez estomacal, mala digestión, "gases", estreñimiento, empacho, frío en el estómago y afecciones de la boca, son las más comunes en la región purépecha (Suárez, 1997; Bello y Salgado, 2007). La familia Asteraceae posee el mayor número de plantas medicinales, seguida de Leguminosae, Lamiaceae, Solanaceae y Rosaceae. Hernández (2005), Bello y Salgado (2007) mencionan la predominancia de Asteraceae con el mayor número de plantas medicinales, seguida por Leguminosae y Lamiaceae. Por lo que se refiere a la porción de la planta más utilizada, la parte aérea (toda la planta) fue la más utilizada, seguida por el tallo, la flor, la hoja y el fruto, las demás partes de la planta como la raíz, la semilla y las ramas fueron las menos utilizadas (cuadro 8).

De las diferentes formas de empleo utizadas, la infusión (45.12\%), la planta aplicada directamente en la zona afectada (2.85\%), el macerado $(2.03 \%)$ y la cataplasma (2.03\%) fueron las más importantes de donde se extraen productos medicinales que son suministrados vía oral, cutánea y local (52.03\%); seguida por el aserrío (9.34\%) y la planta guisada o consumida en fresco $(4.47 \%)$. Las diferentes formas de obtención de las plantas útiles fueron: la colecta con propósitos ceremonial (13.4\%), ornamental (4.47\%), de uso doméstico $(1.62 \%)$ y de uso veterinario $(0.40 \%)$. Otras formas son el pastoreo para la obtención de forraje (11.78\%), plantas melíferas $(1.62 \%)$ y plantas tóxicas para el ganado $(0.81 \%)$.

\section{AGRADECIMIENTO}

Apoyado por el proyecto PIFI/201216MSU0014T-04-01 UMSNH.

\section{Conclusiones}

*Se colectaron 246 especies útiles maderables y no maderables, distribuidas en 59 familias y 135 géneros.

Cuadro 8. Relación entre las parte empleadas y el número especies.

\begin{tabular}{|l|c|c|}
\hline \multicolumn{1}{|c|}{ Parte empleada } & Núm. especies & \% \\
\hline toda la planta (parte aérea) & 155 & 63.00 \\
tallo (leñoso y herbáceo) & 24 & 9.75 \\
flor & 21 & 8.53 \\
hoja & 17 & 6.91 \\
fruto & 16 & 6.50 \\
raíz & 11 & 4.47 \\
semilla & 1 & 0.40 \\
rama & 1 & 0.40 \\
\hline
\end{tabular}


*Los usos registrados fueron: medicinal con 128 especies $(52.03 \%)$, ceremonial con 33 especies (13.41\%), forrajero con 29 especies $(11.78 \%)$, maderable con 23 especies (9.34\%), ornamental y comestible con 11 especies (4.47\%), melífero y de uso doméstico con cuatro especies (1.62\%), tóxico con dos especies $(0.81 \%)$ y de uso veterinario con una especie $(0.40 \%)$.

*La familia que aportó el mayor número de plantas útiles fue Asteraceae con 67 especies $(27.35 \%)$, seguida por las Leguminosae con 23 especies $(9.34 \%)$ y Gramineae con $20(8.13 \%)$.

*Los géneros que aportaron la mayor cantidad de especies útiles fueron: Quercus, Senecio, Salvia, Gnaphalium, y Eupatorium.

*El estrato herbáceo fue la forma más usada para uso medicinal y forrajero principalmente, seguida por el estrato arbustivo y arbóreo.

*De las partes utilizadas, las de mayor uso fue la planta completa, seguida por el tallo, la flor, la hoja para medicina y madera principalmente.

*Las formas de uso y obtención preferidas fueron la infusión, de donde se extraen productos medicinales, seguido por la colecta de plantas con fines ceremoniales, ornamentales, comestibles, de uso doméstico y veterinario y el pastoreo para forraje.

\section{LITERATURA CITADA}

Alarcón-Cháires, P., 2001. Ecología y trasformación campesina en la Meseta P'urhepecha. Universidad Michoacana de San Nicolás de Hidalgo. 261 pp.
Alvarado S., M.L., 1999. "Panorama Fitoterapéutico de Santa Clara del Cobre, Michoacán, México". Tesis profesional. Facultad de Biología. UMSNH. Morelia, Mich. México. 108 pp.

Bello-González, M.A., 1993. Plantas útiles no maderables de la Sierra Purépecha, Michoacán. México. Foll. Tec. Núm. 10. CIRPA-Michoacán. INIFAP. Uruapan. Michoacán. México. $115 \mathrm{pp}$.

Bello-González, M.A., y J.N. Labat, 1987. Los encinos (Quercus) del Estado de Michoacán. INIFAP-CEMCA. Collection Études Mésoaméricaines. Serie 11-9. Cuaderno de Estudios Michoacanos. México, DF. 98 pp.

Bello-González, M.A., y R. Salgado-Garciglia. 2007. "Plantas medicinales de la Comunidad Indígena Nuevo San Juan Parangaricutiro, Michoacán, México”. Biológicas, 9: 126-136.

Bello-González, M.A., y X. MadrigalSánchez. 1996. Estudio florístico del Campo Experimental "Barranca del Cupatitzio”, Uruapan, Michoacán. Folleto Científico Núm. 2. CIRPACMichoacán. INIFAP. Uruapan, Michoacán. México. 48 pp.

Bernal-P., M., 2002. "Estudio florístico y ecológico de las arvenses en cultivos de maíz de temporal de Villa Morelos, Michoacán. México". Tesis profesional. Facultad de Biología. UMSNH. Morelia, Michoacán. México. 44 pp.

Caballero J., y C. Mapes, 1985. "Gathering and subsistence patterns among the 
Bello González, M.A. et al.: Plantas útiles de la comunidad indígena Nvo. Sn. Juan Parangaricutiro, Mich., Méx.

Purhépecha indians of Mexico". Journal of Enthnobiology, 5(1): 31-47.

Carranza, G.E., 2005. “Angiospermas”. pp. 73-75. La biodiversidad en Michoacán (G. L. Villaseñor, editora). Comisión Nacional para el Conocimiento y Uso de la Biodiversidad, Secretaría de Urbanismo y Medio Ambiente, Universidad Michoacana de San Nicolás de Hidalgo. México.

Cotton, C., 1996, Ethnobotany principles and applications. John Wiley and Sons, Nueva York.

Delgado-L., A.M., 2003. "Las plantas medicinales de Cutzato, municipio de Acuitzio del Canje, Michoacán: Uso tradicional, disponibilidad y extracción”. Tesis profesional. Facultad de Biología-UMSNH. Morelia, Michoacán. México. 75 pp.

Díaz-Barriga H., y M.A. Bello-González, 1993. Contribución al conocimiento de la flora de la Cuenca del lago de Pátzcuaro. Libro Técnico Núm. 1. CIRPAC-Michoacán. INIFAP-Uruapan, Michoacán. México. 161 pp.

D.T.F.S.J.N.P., 1988. Estudio de Manejo Integral de Recursos Forestales. Nuevo San Juan Parangaricutiro. Uruapan, Mich., México. 86 pp.

Esparza-G., A., 1989. "La flora útil silvestre no maderable del municipio de Cherán, Michoacán (México)". Tesis profesional. Escuela de Biología-UMSNH. Morelia, Michoacán. México. 110 pp.
Farfán, H.B., 2001. “Aspectos ecológicos y etnobotánicos de los recursos vegetales de la comunidad Mazahua Francisco Serrano, municipio de Zitácuaro, Michoacán, México". Tesis profesional. Facultad de Biología-UMSNH. Morelia, Michoacán. México. 91 pp.

Flores, R., 1987. "Plantas medicinales de la localidad del Cerrito de Cotijarán, Michocán”. Tesis profesional. Esc. de Biología-UMSNH. Morelia, Michoacán. México. 87 pp.

García, E., 1988. Modificaciones al sistema de clasificación climática de Koppen. Universidad Nacional Autónoma de México, DF, 220 pp.

García-L., E., 1990. "Las compuestas silvestres del Valle de Morelia". Tesis profesional. Escuela de Biología-UMSNH. Morelia, Michoacán. México. 247 pp.

García-M., C., 2013. Las repúblicas purépechas. Ed. Electrónicas para internet, México. 22 pp. En formato PDF. http// tsimarhutsimarhu.blogspot.mx/

García-P., J., 2002. "Plantas útiles de San Pedro Jacuáro municipio de Ciudad Hidalgo, Michoacán. México". Tesis profesional. Facultad de BiologíaUMSNH, Morelia, Michoacán. México. $66 \mathrm{pp}$.

Hernández-M., S., 2005. "Flora útil no maderable de la Comunidad Indígena Nuevo San Juan Parangaricutiro, Michoacán. México". Tesis profesional. 
Facultad de Agrobiología-UMSNH, Uruapan, Michoacán. México. 82 pp.

Hurtado-R., N., y J.C. Rodríguez, 2001. Estudio de la flora medicinal del municipio de Copándaro de Galeana, Michoacán, México. XV Congreso Mexicano de Botánica, Querétaro, Qro., México. Resumen 644.

Instituto Nacional de Estadística, Geografía e Informática, 1985. Síntesis Geográfica de Michoacán. Secretaría de Programación y Presupuesto. México.

Madrigal-Sánchez, X., 1992. "Los bosques michoacanos: su conocimiento y conservación". 1 Muestra. Los recursos vegetales de Michoacán. Memoria. Morelia, Mich. México. pp. 65-74.

Martínez-R., M., 1997. "Contribución al conocimiento de las plantas vasculares en los bosques de la Comunidad Indígena de Nuevo San Juan Parangaricutiro, Michoacán, México". Tesis profesional. Facultad de Biología-UMSNH. Morelia, Michoacán, México. 67 pp.

Martín, G.J., 2004. Ethnobotany: a methods manual. Earthscan Publications. 268 pp.

Mas, J.F.; A. Velazquez, J. R. Díaz-Gallegos, R. Mayorga-Saucedo, C. Alcántara, G. Bocco, R. Castro, T. Fernández, y A. Pérez-Vega, 2004. “Assessing land use/cover changes: a nationwide multidate spatial database for Mexico". International Journal of Applied Earth Observation and Geoinformation.
Medina, C.; F.F. Guevara, M.A. Martínez, S. Silva, M.A. Chávez-Carbajal, e I. García, 2000. "Estudio Florístico en el área de la Comunidad Indígena de Nuevo San Juan Parangaricutiro, Michoacán. México". Acta Botánica Mexicana, 52: 5-41.

Norma Oficial Mexicana NOM-059-SEMARNAT-2001. "Protección ambiental-Especies nativas de México de flora $\mathrm{y}$ fauna silvestres-Categorías de riesgo y especificaciones para su inclusión, exclusión o cambio-Lista de especies en riesgo". Diario Oficial de la Federación. 6 de marzo del 2002.

Page, J.T.P., 1995. "Health Policy and Legislation Concerning Traditional Indigenous Medicine in Mexico". Cad. Saúde Públ., Rio de Janeiro, 11(2): 201-211.

Pérez-G., A.L.; A.M. Rosales., M. Maya, y J.L. Arias, 1994. "Contribución al conocimiento de las plantas medicinales en Maruata, Michoacán, México". Revista Trimestral. Facultad de Biología-UMSNH. 14: 55.

Rojas-C., M.G., 2006. "Plantas medicinales de Caltzontzin, municipio de Uruapan, Michoacán". Tesis profesional. Facultad de Biología-UMSNH. Morelia Michoacán. México. 90 pp.

Rzedowski, J., 1991. "Diversidad, orígenes de la flora fanerogámica de México. Instituto de Ecología". Centro Regional del Bajío, Pátzcuaro, Michoacán. México. Acta Bot. Méx., 14: 3-21. 
Bello González, M.A. et al.: Plantas útiles de la comunidad indígena Nvo. Sn. Juan Parangaricutiro, Mich., Méx.

Sepúlveda, M.T., 1996. "La medicina entre los Purépechas prehispánicos". P. 113-134. Plantas medicinales de México (Erick Estrada Lugo, Editor). Universidad Autónoma de Chapingo, Texcoco. México.

Silva, S.P., 1996. "Inventario de la flora vascular y de plantas útiles de los pedregales del municipio de Huaniqueo, Michoacán, México". Tesis profesional, Facultad de Biología-UMSNH. Morelia, Michoacán. México. 26 pp.
Suárez, C., 1997. Manual para el uso de las plantas medicinales en la región del Lago de Pátzcuaro. Centro de Estudios Sociales y Ecológicos. Pátzcuaro, Mich. 166 pp.

Torres-R., L., 1994. “Contribución al conocimiento de las plantas vasculares del cerro Patamban, municipio de Tangancícuaro y Los Reyes Michoacán, México". Tesis profesional. Facultad de Biología-UMSNH. Morelia, Michoacán. México. 132 pp.

Recibido: 20 agosto 2013. Aceptado: 23 junio 2014. 


\section{Apéndice}

Listado de plantas útiles de la Comunidad Indígena Nuevo San Juan Parangaricutiro, Michoacán.

\section{PTERIDOPHYTA}

\section{ASPLENIACEAE}

Asplenium monanthes L. $(1,2)$ "helecho". "pescadillo". PARTE UTILIZADA: toda la planta. USO: ornamental y ceremonial. FORMA DE EMPLEO: en macetas.

\section{DENNSTAEDTIACEAE}

Pteridium aquilinum (L.) Kuhn. (4, 5, 6, 8, $11,12,13,16,17,19,21,23,26,27,29,30$, 32) "pescadillo" "palmillo". PARTE UTILIZADA: raíz, tallo, toda la planta. USO: medicinal (quemaduras, dolor de muelas), doméstico. FORMA DE EMPLEO: se frota el tallo o la raíz en la parte quemada; se prepara el cocimiento de un puño de raíz en un litro de agua y se hacen buches o se aplicada localmente; con la parte aérea se elaboran estropajos.

\section{POLYPODIACEAE}

Polypodium alasmithii R. C. Moran. PARTE UTILIZADA: toda la planta. USO: ceremonial, ornamental. FORMA DE EMPLEO: con la parte aérea adornan altares (Hernández, 2005).

Polypodium plebeium Schlecht. \& Cham. $(3,4,5,8,17)$ "kurucha-arimbikua", "pescado arhikata". PARTE UTILIZADA: toda la planta. USO: medicinal (contra la epilepsia). FORMA DE EMPLEO: baños con la cocción de la planta.

PTERIDACEAE

Adiantum andicola Liebm. (4, 9, 12, 15,
18, 28, 27, 29) "cilantrillo", "kulandu". PARTE UTILIZADA: toda la planta. USO: medicinal (empacho, posparto, estreñimiento infantil), ornamental. FORMA DE EMPLEO: se prepara un cocimiento de una taza de la planta en medio litro de agua, se toma tres veces al día o como agua de uso; en macetas.

Adiantum raddianum Presl. Tent. PARTE UTILIZADA: toda la planta. USO: ornamental y ceremonial: FORMA DE EMPLEO: en macetas y adorno de altares (Hernández, 2005).

Adiantum tenerum Swartz. PARTE UTILIZADA: toda la planta. USO: ornamental. FORMA DE EMPLEO: en macetas (Hernández, 2005).

\section{GYMNOSPERMAE}

\section{CUPRESSACEAE}

Cupressus lindleyi Klotzsch ex Endl. (1, 6, 15) "cedro blanco". PARTE UTILIZADA: rama. USO: medicinal (tos, dolor de oídos, padecimientos de la mujer). FORMA DE EMPLEO: se toma el cocimiento de un pedacito de rama mezclada con canela en medio litro de agua, se toman tres tazas al día hasta que ceda la tos; se pone a macerar un pedazo de rama en medio litro de alcohol durante 24 horas, se aplica externamente; se toma el cocimiento de las hojas mezcladas con canela.

\section{PINACEAE}

Abies religiosa Schl. \& Cham. (1, 6, 15, 17, $18,19)$ "oyamel", "pinabete". PARTE UTILIZADA: tronco. USO: maderable (leña, aserrío, construcciones rurales, pulpa para papel), ceremonial (adorno para altares), medicinal (padecimientos de la piel, siste- 
Bello González, M.A. et al.: Plantas útiles de la comunidad indígena Nvo. Sn. Juan Parangaricutiro, Mich., Méx.

ma circulatorio y digestivo) FORMA DE EMPLEO: madera aserrada y/o en astilla; se aplica la resina o se mezcla con resina de pino, inguambo (Bocconia arborea).

Pinus leiophylla Sch. \& Cham. (3, 5, 6, $7,8,11,15,20)$ "pino chino", "pino prieto". PARTE UTILIZADA: tronco. USO: maderable (construcciones, duela, leña y postes para cerca). FORMA DE EMPLEO: madera aserrada y sin labrar.

Pinus michoacana var. cornuta Mart. \& Gal. $(5,7,20)$ "pino lacio". PARTE UTILIZADA: tronco. USO: maderable (construcciones rurales y urbanas, durmientes, cimbras, aserrío y leña), carbón, resina. FORMA DE EMPLEO: madera aserrada y sin labrar.

Pinus montezumae Lamb. (5, 6, 7, 13,17) "pino prieto". PARTE UTILIZADA: tronco. USO: maderable (construcciones rurales y urbanas, leña, carbón, resina). FORMA DE EMPLEO: madera aserrada y sin labrar.

Pinus pseudostrobus Schl. \& Cham. (2, 3, $5,6,19)$ "pino cantzimbo". PARTE UTILIZADA: tronco. USO: maderable (madera aserrada para la fabricación de muebles). FORMA DE EMPLEO: madera aserrada.

\section{MONOCOTYLEDONEAE}

COMMELINACEAE

Commelina tuberosa L. $(8,13,14)$ "zapatillo", "yuca", "clavelillo", "jicamilla", "platanillo", "uarhasi". PARTE UTILIZADA: raíz. USO: medicinal (Aire en los oídos). FORMA DE EMPLEO: la raíz machacada se coloca en forma de tapón en los oídos.
Commelina coelestis Willd. $(5,6,7,10)$ "hierba del pollo", "empanadilla". PARTE UTILIZADA: raíz. USO: medicinal (Afecciones del riñón). FORMA DE EMPLEO: se prepara una infusión con media taza de planta en un litro de agua, se toma como agua de usos durante el día.

Tripogandra purpurascens (Schauer) Hadlos. $(5,6,9,13,19)$ "platanillo", "Moradilla", "yuchi-arimbikua". PARTE UTILIZADA: toda la planta. USO: medicinal (afecciones del riñón). FORMA DE EMPLEO: se prepara una infusión con media taza de planta en un litro de agua, se toma máximo cuatro tazas durante el día.

\section{CYPERACEAE}

Cyperus hermaphroditus (Jacq.) Standl. "grama gruesa". PARTE UTILIZADA: toda la planta. USO: ceremonial, forrajera. FORMA DE EMPLEO: la parte aérea es utilizada en adornos para altares; pastoreo cerril (Hernández, 2005).

Cyperus spectabilis Link. (4, 7, 14, 16). PARTE UTILIZADA: toda la planta. USO: forrajera, ornamental. FORMA DE EMPLEO: pastoreo cerril. Adornos.

\section{IRIDACEAE}

Sisyrinchium tenuifolium H \& B Ex. Willd. "flor cimarrona" "primavera". PARTE UTILIZADA: toda la planta. USO: ceremonial. FORMA DE EMPLEO: con la parte aérea adornan coronas para el día de muertos (Hernández, 2005).

Sisyrinchium schaffneri Watson. (16) "zacate", "tup'iku”. PARTE UTILIZADA: toda la planta. USO: medicinal (contra parásitos intestinales). FORMA DE EMPLEO: hervida y tomada como té. 


\section{LILIACEAE}

Smilax moranensis Mart, \& Gal. (3, 11, 26, 28) "mecate de zorra" "zarzaparrilla". PARTE UTILIZADA: toda la planta. USO: medicinal (purificar la sangre), ceremonial. FORMA DE EMPLEO: se prepara la infusión de un puño de la planta en medio litro de agua, se toma como agua de uso; con la parte aérea se adornan coronas para el día de muertos.

\section{ORCHIDACEAE}

Govenia liliaceae (Lex.) Lindl. (16) "lirio", "tsitsi inchamakurhi". PARTE UTILIZADA: toda la planta. USO: ornamental. FORMA DE EMPLEO: arreglos florales; colecta.

\section{POACEAE}

Aegopogon cenchroides Humb. \& Bonpl. (4) "grama del cerro", "jarauten", "cuitisil", "huachacha", "uitsaku pirikata, uitsaku xugapiti". PARTE UTILIZADA: hoja, ta1lo. USO: forrajera, doméstico, medicinal (enfermedades de los riñones). FORMA DE EMPLEO: pastoreo cerril; elaboración de estropajos y nidos para gallinas de postura; hervida con flor de alfalfa (Medicago sativa) y tomada como agua de uso.

Aegopogon tenellus (DC.) Trin. "grama delgada" "zacate colorado". PARTE UTILIZADA: toda la planta. USO: ornamental, forrajera. FORMADE EMPLEO: la parte aérea una vez seca se pinta y se utiliza como naturaleza muerta; pastoreo cerril (Hernández, 2005). Agrostis hyemalis (Walt.) B.S.P. (4) "liendrilla", "xundi sapichu". PARTE UTILIZADA: toda la planta. USO: forrajera. FORMA DE EMPLEO: pastoreo cerril.

Bouteloua curtipendula (Michx.) Torr. (4) "banderilla", "grama del cerro", "uitsaku juatarhu". PARTE UTILIZADA: toda la planta. USO: forrajera. FORMA DE EMPLEO: pastoreo cerril.

Bromus carinatus Hook. \& Arn. $(14,15)$ "tupikua", "tigrillo", "grama", "camaloti”, "zacate". PARTE UTILIZADA: toda la planta. USO: forrajera. FORMA DE EMPLEO: pastoreo cerril.

Bromus dilichocarpus Wagnon. (5, 6, 7, 11, $16,17,21)$ "carantacuz", "ballico". PARTE UTILIZADA: toda la planta. USO: forrajera, medicinal (padecimiento de la piel, fiebre). FORMA DE EMPLEO: pastoreo cerril; con el cocimiento de la parte aérea de la planta se baña al enfermo.

Digitaria leucites (Trin.) Henrard. $(14,15)$ "grama blanca", "liendrilla", "zacate de caballo", "uitsaku urapiti xundi", "tupikua xanxankiri". PARTE UTILIZADA: raíz, toda la planta. USO: doméstico. FORMA DE EMPLEO: sirve para amarrar el rastrojo; pastoreo cerril.

Echinochloa oplismenoides (HBK.). Chase. $(14,15)$. PARTE UTILIZADA: toda la planta. USO: forrajera, ornamental. FORMA DE EMPLEO: pastoreo cerril; la parte aérea una vez seca se pinta y se utiliza como naturaleza muerta.

Festuca callosa (Piper) Saint-Yves. "avena". PARTE UTILIZADA: toda la planta. USO: forrajera. FORMA DE EMPLEO: pastoreo cerril. (Hernández, 2005).

Festuca amplissima Rupr. PARTE UTILIZADA: toda la planta. USO: forrajera. FORMA DE EMPLEO: pastoreo cerril. (Hernández, 2005). 
Bello González, M.A. et al.: Plantas útiles de la comunidad indígena Nvo. Sn. Juan Parangaricutiro, Mich., Méx.

Lasiacis nigra Davidse. (14,15) "itzimarimbicua", "agatapu siruku". PARTE UTILIZADA: toda la planta. USO: forrajera. FORMA DE EMPLEO: pastoreo cerril.

Muhlenbergia macroura (H.B.K.) Hitchc.(18) "tzurumuta", "palmilla". PARTE UTILIZADA: toda la planta. USO: forrajera. FORMA DE EMPLEO: pastoreo cerril.

Muhlenbergia fragilis Swallen. "liendrilla". PARTE UTILIZADA: toda la planta. USO: forrajera, medicinal (enfermedades del aparato digestivo) FORMA DE EMPLEO: pastoreo cerril; se toma el cocimiento de la parte aérea (Hernández, 2005).

Muhlenbergia robusta (Fourn.) Hitchc. (18) "tzurumuta", "espiga", "pintsi". PARTE UTILIZADA: toda la planta. USO: forrajera. FORMA DE EMPLEO: pastoreo cerril.

Paspalum notatum Flugge. (18) "hierba de la vibora". PARTE UTILIZADA: toda la planta. USO: medicinal (padecimientos de la piel, golpes). FORMA DE EMPLEO: con el cocimiento de la parte aérea de la planta se aplican fomentos en la parte afectada.

Paspalum squamulatum Fourn. (18) "tigrillo", "amatsi". PARTE UTILIZADA: toda la planta. USO: forrajera, FORMA DE EMPLEO: pastoreo cerril.

Piptochaetium fimbriatum (H.B.K.) Hitchc. $(8,13)$ "zacate", "tupiku”. PARTE UTILIZADA: toda la planta. USO: medicinal (diarrea y vómito), forrajera. FORMA DE EMPLEO: se toma el cocimiento de la parte aérea; pastoreo cerril.
Piptochaetium virescens (H.B.K.) Parodi. (3) "popotillo", "takarin", "ak'arhini". PARTE UTILIZADA: toda la planta. USO: forrajera. FORMA DE EMPLEO: pastoreo cerril.

Sporobolus indicus (L.) R. Br. (3) "tzurumuta", "liendrilla", "palmilla". PARTE UTILIZADA: raíz. USO: doméstico, forrajera. FORMA DE EMPLEO: se elaboran escobas y se amarra el rastrojo; pastoreo cerril.

Trisetum virletii Fourn. "tabardillo del cerro". PARTE UTILIZADA: toda la planta. USO: ceremonial FORMA DE EMPLEO: con la parte aérea se adornos los altares (Hernández, 2005).

\section{DICOTYLEDONEAE \\ AMARANTHACEAE}

Amaranthus hybridus L. "quelite". PARTE UTILIZADA: toda la planta. USO: comestible. FORMA DE EMPLEO: se consumen las hojas tiernas guisadas (Hernández, 2005).

Iresine diffusa Humb. \& Bonpl. ex Willd. $(4,12,13,17,19,21)$ "hierba del arlomo" "hierba del clavo". PARTE UTILIZADA: toda la planta. USO: medicinal (Padecimientos de la piel), ceremonial. FORMA DE EMPLEO: con el cocimiento de la parte aérea se aplica en fomentos; con la parte aérea se adornan altares.

\section{AQUIFOLIACEAE}

Ilex pringlei Standl. (12) "bola negra". PARTE UTILIZADA: tronco. USO: maderable (artesanías, elaboración de collares y pulseras). FORMA DE EMPLEO: madera aserrada. 


\section{ASTERACEAE}

Ageratum corymbosum Zuccagni. (12, 17, 19) "servilletilla". PARTE UTILIZADA: raíz. USO: medicinal (afecciones del riñón, biliar). FORMA DE EMLEO: se toma el cocimiento de una taza de raíz en dos litros de agua, se toma una taza tres veces al día.

Artemisia ludoviciana Nutt. (19) "estafiate". PARTE UTILIZADA: toda la planta. USO: medicinal (dolor de estómago). FORMADE EMPLEO: se prepara el cocimiento de un puño de estafiate en un litro de agua, se toma una taza tres veces al día.

Archibaccaris hirtella (DC.) Heering. (17, 19) "garañona". PARTE UTILIZADA: toda la planta. USO: medicinal (padecimientos de la mujer). FORMA DE EMPLEO: se toma el cocimiento de la parte aérea (Hernández, 2005).

Astranthium orthopodum B.L. Rob. \& Fernald. (1, 8, 9, 11, 15, 17, 19, 22, 29, 32) "bola de hilo", "árnica blanca". PARTE UTILIZADA: toda la planta. USO: medicinal (hemorragias de parto, cólico, tos y ulceras, piel), forrajera, melífera. FORMA DE EMPLEO: hervida y tomada como te; pastoreo cerril

Baccharis conferta HBK. "karatacua". PARTE UTILIZADA: toda la planta. USO: doméstico. FORMA DE EMPLEO: con la parte aérea se elaboran escobas (Hernández, 2005).

Baccharis heterophylla HBK. (26, 27, 29, 30, 31, 32 "hierba de la mula" "karatacua". PARTE UTILIZADA: toda la planta, raíz. USO: medicinal (enfermedades del aparato digestivo y urinario), doméstico. FORMA DE EMPLEO: se elaboran tinturas y se toma; con el cocimiento de la raíz se enjuagan la boca; con la parte aérea se elaboran escobas (Hernández, 2005).

Baccharis pteronioides DC. (1) "karatacua". PARTE UTILIZADA: toda la planta. USO: medicinal (diabetes, dolor de muelas), doméstico. FORMA DE EMPLEO: se prepara el cocimiento de una taza de la planta en un litro de agua y se toma una taza tres veces al día; con la parte aérea se elaboran escobas.

Bidens aequisquama (Fernald.) Sherff. $(3,4,7,11,15)$ "moradilla", "amapola", "aceitilla". PARTE UTILIZADA: toda la planta, raíz, flor. USO: medicinal (para cólicos y tos), forrajera, melífera, ornamental. FORMA DE EMPLEO: hervida con flor de "tabachin", "guayacan" y tomada como té; masticada, pastoreo cerril; en floreros.

Bidens aurea (Ait.) Sherff. (4) "aceitilla", "kutsumu-tzupambiti", "aceitilla amari1la”. PARTE UTILIZADA: toda la planta. USO: medicinal (dolor de pecho y para purificar la sangre), forrajera y ornamental. FORMA DE EMPLEO: hervida y tomada como té; pastoreo cerril; en floreros.

Bidens ferulifolia (Jacq.) DC. (9, 10, 14, 18) "aceitilla", "amapolita". PARTE UTILIZADA: toda la planta. USO: medicinal (enfermedades del aparato respiratorio), ceremonial. FORMA DE EMPLEO: con el cocimiento de la parte aérea se toman tres tazas al día; con la parte aérea adornan coronas el día de muertos.

Bidens odorata Cav. $(1,5,6,8,10,12$, $13,14,15,17,18,19,20,21)$ "aceitilla blanca", "kutsumu urapiti. PARTE UTILIZADA: toda la planta. USO: medicinal 
(contra diarrea, broquitis y tos), forrajera, ceremonial, ornamental. FORMA DE EMPLEO: hervida con canela y tomada como té; pastoreo cerril; en las coronas del día de muertos y en floreros.

Bidens ostruthioides (DC.). ( 4, 6, 7, 11, 12, $15,17,21)$ "aceitilla", "kutzumu”. PARTE UTILIZADA: toda la planta. USO: medicinal (padecimientos de la piel), ceremonial. FORMA DE EMPLEO: con el cocimiento de la parte aérea se baña al enfermo; con la parte aérea adornan coronas para el día de muertos

Bidens triplinervia H.B.K. $(11,15)$ "aceitilla", "Kutsumu". PARTE UTILIZADA: flor. USO: melífera.

Calea scabra Lag. (3, 10) "quien sabe", "nendi miti", "jauandi”. PARTE UTILIZADA: toda la planta. USO: medicinal (contra el vómito), forrajera, ceremonial. FORMA DE EMPLEO: hervida con sal y tomada como té; pastoreo cerril; con las flores adornan altares.

Cirsium acantolepis (Hemsl.) (3, 10) “cardo santo". PARTE UTILIZADA: toda la planta, flor. USO: medicinal (cálculos biliares, tos ferina y pulmones). FORMA DE EMPLEO: se toma el cocimiento de un puñado de la planta o de la flor en un litro con agua.

Cirsium anartiolepis Petrak. (1) "cardo santo", "xukurhi aparhiku”. PARTE UTILIZADA: toda la planta. USO: medicinal (enfermedades del corazón). FORMA DE EMPLEO: hervida y tomada como té.

Cirsium ehrenbergii Sch. Bip. (18) "cardo santo". PARTE UTILIZADA: flor. USO: medicinal (enfermedades del sistema respiratorio). FORMA DE EMPLEO: se toma el cocimiento de la flor.

Cirsium subcoriaceum (Less.). Sch. Bip. (1) "cardo santo", "xukurhi aparhiku". PARTE UTILIZADA: toda la planta. USO: medicinal (enfermedades del corazón). FORMA DE EMPLEO: hervida y tomada como té.

Conyza schiedeana Less. (8, 9, 14, 15, 17, 20) "simonillo", "gordolobo", "xurhatajasi”. PARTE UTILIZADA: toda la planta. USO: medicinal (antibilioso), forrajera. FORMA DE EMPLEO: hervida y tomada como té; pastoreo cerril.

Erigeron delphinifolius Willd. (8) "árnica blanca", “arnika urapiti”. PARTE UTILIZADA: toda la planta. USO: ceremonial, ornamental, forrajera, medicinal (golpes). FORMA DE EMPLEO: en coronas y festejos religiosos del día de muertos; en floreros; pastoreo cerril; macerada y en cataplasmas.

Eupatorium areolare DC. (17) "borreguilla", "karichi sapichu agatapu". PARTE UTILIZADA: hoja. USO: medicinal (contra enfermedades biliosas), forrajera. FORMA DE EMPLEO: hervida y tomada como té.

Eupatorium arsenei Rob. "vara blanca" "toqueri". PARTE UTILIZADA: toda la planta. USO: ceremonial, forrajera. FORMA DE EMPLEO: con la parte aérea adornan altares y coronas para el día de muertos; pastoreo cerril. (Hernández, 2005).

Eupatorium glabratum HBK. (3, 22, 24, 25, 27, 29) "hierba de la mula", "mula 
xukurhi". PARTE UTILIZADA: toda la planta. USO: medicinal (golpes, fracturas), forrajera, doméstico, melífera. FORMA DE EMPLEO: macerada o machacada y en cataplasmas; pastoreo cerril; la flor es visita por las abejas.

Eupatorium mairetianum DC. "toqueri". PARTE UTILIZADA: toda la planta. USO: ceremonial, forrajera. FORMA DE EMPLEO: la parte aérea es utilizada en adornos para altares; pastoreo cerril (Hernández, 2005)

Eupatorium pazcuarense HBK. "hierba del burro". PARTE UTILIZADA: toda la planta. USO: ceremonial, ornamental. FORMA DE EMPLEO: con la parte aérea se adornan altares y coronas para el día de muertos; la parte aérea es utilizada en adornos florales (Hernández, 2005).

Eupatorium pycnocephalum Less. PARTE UTILIZADA: toda la planta. USO: ceremonial. FORMA DE EMPLEO: con la parte aérea adornan altares (Hernández, 2005).

Eupatorium schaffneri Sch. Bip. ex B.L. Rob. PARTE UTILIZADA: toda la planta. USO: ceremonial. FORMA DE EMPLEO: con la parte aérea adornan los altares (Hernández, 2005).

Galinsoga quadriradiata Ruiz \& Pavón. PARTE UTILIZADA: toda la planta. USO: ceremonial. FORMA DE EMPLEO: con la parte aérea adornan altares (Hernández, 2005).

Gnaphalium americanum Mill. (1, 3, 6, 7, 8, 10, 11, 13, 15, 17, 20) "gordolobo", "xurhateri". PARTE UTILIZADA: toda la planta. USO: medicinal (expectorante, golpes). FORMA DE EMPLEO: hervida y tomada como té.

Gnaphalium attenuatum DC. (9) "gordolobo", "xurhateri". PARTE UTILIZADA: toda la planta. USO: medicinal (contra la tos). FORMA DE EMPLEO: hervida con canela y tomada como té.

Gnaphalium bourgovii Gray. (9) "gordolobo", PARTE UTILIZADA: toda la planta. USO: medicinal (contra la tos). FORMA DE EMPLEO: hervida con canela y leche y tomada (Hernández, 2005).

Gnaphalium inornatum DC. $(3,4)$ "gordolobo", "xurhateri". PARTE UTILIZADA: toda la planta. USO: medicinal (contra la tos y dolor de estómago). FORMA DE EMPLEO: hervida con canela y tomada como té.

Gnaphalium liebmannii SCH. Bip. Ex Klatt. (8, 14, 18) "gordolobo". PARTE UTILIZADA: toda la planta. USO: medicinal (contra la tos). FORMA DE EMPLEO: hervida con canela o grama y tomada como té.

Gnaphalium oxyphyllum DC. $(3,4)$ "gordolobo", "xurhateri". PARTE UTILIZADA: flor. USO: medicinal (contra la tos). FORMA DE EMPLEO: hervida con corteza de chirimoya (Annona cherimola), árnica (Heterotheca inuloides) y flor de camelina (Bougainvillea sp.) y tomada como té.

Gnaphalium salicifolium (Bertol.) DC. (9) "gordolobo". PARTE UTILIZADA: toda la planta. USO: medicinal (contra la tos). FORMA DE EMPLEO: se toma el cocimiento de la parte aérea.

Gnaphalium semiamplexicaule DC. (3, 11, $17,20)$ "gordolobo". PARTE UTILIZA- 
DA: toda la planta. USO: medicinal (afecciones del aparato respiratorio). FORMA DE EMPLEO: se toma el cocimiento de la parte aérea, se endulza con miel o se mezcla con "arnica" (Heterotheca inuloides), flor de "camelina" (Bougainvillea sp.), "hierba del sapo" (Eryngium spp.), o "espinosilla" (Loeselia mexicana).

Heterotheca inuloides Cass. (1, 2, 4, 5, $6,7,11,12)$ “árnica”, "arnika xukurhi”. PARTE UTILIZADA: flor, toda la planta. USO: medicinal (golpes, reumatismo, tos). FORMA DE EMPLEO: macerada y untada, hervida con canela, miel, hojas de tomate y tomada como té.

Jaegeria hirta (Lag.) Less. (3, 4, 13, 14, 19) "panalillo", "kuipu sapichu”. PARTE UTILIZADA: toda la planta. USO: forrajera. FORMA DE EMPLEO: pastoreo cerril.

Melampodium perfoliatum Cav. $(7,8)$ "segundo andan", "andan", “quesitos", "andani ketsikua". PARTE UTILIZADA: toda la planta. USO: forrajera. FORMA DE EMPLEO: pastoreo cerril.

Perymenium berlandieri DC. PARTE UTILIZADA: toda la planta. USO: Forrajera. FORMA DE EMPLEO: pastoreo cerril (Hernández, 2005).

Perezia michoacana B.L. (3, 9, 10) “clavelillo", "klabeli agatapu". PARTE UTILIZADA: flor, toda la planta. USO: medicinal (contra enfermedades del riñón), forrajera. FORMA DE EMPLEO: hervida y tomada como agua de uso; pastoreo cerril.

Piqueria pilosa HBK. "tabardillo blanco" PARTE UTILIZADA: toda la planta. USO: ceremonial. FORMA DE EMPLEO: con la parte aérea adornan altares y coronas para el día de muertos (Hernández, 2005).

Piqueria triflora Hemsl. (9, 14, 15, 17) “tabardillo", "kuini-kumanchikua", "sombra de pájaro". PARTE UTILIZADA: raíz, flor. USO: medicinal (antidiarreico, empacho, fiebre intestinal, hemorragias vaginales), ceremonial, veterinario. FORMA DE EMPLEO: hervida con hojas de "fresno" (Fraxinus), cinco limones y cuatro cucharadas de sal y tomada como té; después del parto molida y untada; se utiliza en coronas del día de muertos; hervida y tomada.

Piqueria trinervia Cav. (5, 6, 9, 13, 20) "tabardillo", "hanikua-manchikua", “jarikuakumanchikua”. PARTE UTILIZADA: toda la planta, flor, tallo. USO: medicinal (contra la fiebre, cólicos y antibilioso), ceremonial, ornamental, forrajera y doméstico. FORMA DE EMPLEO: hervida y tomada como té se baña al enfermo; se utiliza en las coronas del día de muertos; en floreros; pastoreo cerril; con los tallos se elaboran juegos pirotécnicos.

Senecio albonervius Greenm. (12) "tsitsiki-achkuri", "zupiach", "parakua-huemba”. PARTE UTILIZADA: flor. USO: ceremonial, ornamental, melífera. FORMA DE EMPLEO: se hacen "limpias"; en floreros.

Senecio angulifolius DC. $(9,15)$ "paratsutsuki", "kojkurha", "mano ancha". PARTE UTILIZADA: toda la planta. USO: ornamental. FORMA DE EMPLEO: en floreros.

Senecio mexicanus McVaugh. $(9,15)$ "vara blanca", "putsukata urapiti". PARTE UTILIZADA: toda la planta. USO: ceremonial, 
ornamental. FORMA DE EMPLEO: para hacer "limpias"; en floreros.

Senecio peltiferus Hemsl. PARTE UTILIZADA: flor. USO: melífera (Hernández, 2005).

Senecio roldana DC. "flor de melón". PARTE UTILIZADA: toda la planta, hojas. USO: ceremonial, doméstico. FORMA DE EMPLEO: la parte aérea es utilizada en adornos para altares; con las hojas se envuelven quesos y corundas (tamal de masa de maíz mezclado con otros ingredientes, platillo típico de la región purépecha) (Hernández, 2005).

Senecio salignus DC. (1, 3, 25, 27, 29. 30, 31) “jara”, “jaktin”, “jara-tokstini”. PARTE UTILIZADA: toda la planta, hoja, flor y ramas. USO: medicinal (cólicos, empacho, fiebre, rabia), forrajera, ceremonial, melífera, doméstico, ornamental. FORMA DE EMPLEO: se prepara en medio litro de agua la infusión de un puño de la planta con cenizas, se toma una taza en ayunas hasta que ceda las molestias; pastoreo cerril; se hacen "limpias" y cataplasmas; con las ramas se limpian los hornos donde se elabora el pan; en floreros.

Senecio sangisorbae DC. PARTE UTILIZADA: flor. USO: ceremonial, FORMA DE EMPLEO: las flores son utilizadas para el adorno de altares (Hernández, 2005).

Senecio sinuatus HBK. $(5,19)$. "flor de meloncillo". PARTE UTILIZADA: flor. USO: medicinal (dolor de estómago). FORMA DE EMPLEO: se prepara en un cuarto de litro de agua la infusión de un puño de flor, se toma una taza cada cuatro horas.
Senecio stoechadiformis DC. (4, 8, 13, $14,15,16,17,19,20,23,26,29,32)$. "juanilla", "cureschme-anapu-tsitsiki", "estafiate-cimarrón”, "xukurhi kame”. PARTE UTILIZADA: flor, hoja, toda la planta. USO: medicinal (antidiarreico, cólicos, "mal de orina"), ceremonial, ornato, forrajera. FORMA DE EMPLEO: hervida y tomada como té; se utilizan en "limpias"; en floreros; pastoreo cerril.

Sigesbeckia jorullensis H.B.K. (11). PARTE UTILIZADA: hoja. USO: medicinal (contra la disentería). FORMA DE EMPLEO: hervida y tomada como té.

Sonchus oleraceus L. (1, 6, 15, 16) "chicoria" "rabanillo". PARTE UTILIZADA: hoja. USO: medicinal (contra el “chincual"(salpullido), diabetes). FORMA DE EMPLEO: hervida y aplicada en forma de cataplasmas o fomentos; tomada como agua de uso.

Stevia monardifolia H.B.K. $(1,4,17)$ "panalillo blanco", "kuipu sapichu urapiti". PARTE UTILIZADA: toda la planta. USO: ornamental. FORMA DE EMPLEO: en floreros.

Stevia origanoides HBK. (8, 11, 12, 14, $15,20)$ "hierba del burro". PARTE UTILIZADA: toda la planta. USO: medicinal (fiebre), ceremonial. FORMA DE EMPLEO: en un litro de agua se coloca media taza de planta en cocimiento, después de 3 minutos de hervor se retira y se tapa, se toma 3 veces al día; la parte aérea es usada en adornos para altares y coronas durante las festividades día de muertos.

Stevia ovata Willd. PARTE UTILIZADA: toda la planta. USO: ceremonial. FORMA 
Bello González, M.A. et al.: Plantas útiles de la comunidad indígena Nvo. Sn. Juan Parangaricutiro, Mich., Méx.

DE EMPLEO: la parte aérea es usada en adornos para altares (Hernández, 2005).

Stevia salicifolia Cav. (4, 10, 14, 15, 18) "hierba del burro" "hierba de la mula" "toqueri”. PARTE UTILIZADA: toda la planta, hoja, raíz. USO: medicinal (padecimientos de la piel, dolores de cabeza, enfermedades del aparato digestivo), ceremonial. FORMA DE EMPLEO: con el cocimiento de la raíz se baña al enfermo; las hojas se aplican en forma de "chiqueadores" (hoja humedecida colocada en la sienes); se toma el cocimiento de la raíz mezclada con aceite de "ricino" (Ricinus communis); la parte aérea es usada en adornos para altares.

Stevia subpubescens Lag. (19) "hierba de la mula". PARTE UTILIZADA: toda la planta. USO: medicinal (torceduras de huesos). FORMA DE EMPLEO: se aplican en forma de cataplasmas.

Tagetes filifolia Lag. (5, 7, 12, 16, 17, 19) "anis", "putsuti". PARTE UTILIZADA: toda la planta. USO: medicinal (cólicos), comestible (como condimento en el atole de maíz), medicinal (cólicos). FORMA DE EMPLEO: hervida y tomada como té; colecta.

Tagetes foetidissima DC. (4, 8, 9, 10, 11, 14, 15, 18) "cincollaga" "cempasúchil". PARTE UTILIZADA: toda la planta. USO: medicinal (enfermedades de los sistemas circulatorio, nervioso y del aparato digestivo). FORMA DE EMPLEO: se toma el cocimiento de la flor mezclado con manzana; se toma el cocimiento de la planta completa se endulza con piloncillo o se mezcla con "hierba buena".
Tagetes lunulata Ort. (1, 12, 13, 17, 19, 21) "cincollaga". PARTE UTILIZADA: toda la planta. USO: medicinal (enfermedades de los sistemas circulatorio, nervioso y del aparato digestivo), ceremonial. FORMA DE EMPLEO: se toma el cocimiento de la raíz; se toma el cocimiento de la planta completa mezclada con raíz de "lucema" (Salvia lavanduloides) y "cenicilla" (Helianthemum glomeratum); la parte aérea es utilizada para el adorno de altares

Tagetes micrantha Cav. (4, 8, 13) "anis", anisillo", "putsuti". PARTE UTILIZADA: toda la planta. USO: comestible (Como condimento en el atole de maíz), medicinal (cólicos). FORMA DE EMPLEO: colecta; hervida y tomada como té.

Tagetes remotiflora Kunze. (4) "cincollaga", "tiringe", "cempasuchili", "flor de muerto amarillo". PARTE UTILIZADA: raíz, toda la planta. USO: medicinal (antidiarreico, contra el vómito, nervios, sofocación, presión arterial, partos), ornamental. FORMA DE EMPLEO: hervida con canela tomada como té; hervida con chocolate ayuda a facilitar el parto; en adornos.

Tithonia tubaeformis (Jacq.) Cass. (13, 17, 19) "andan". PARTE UTILIZADA: flor. USO: medicinal (enfermedades del aparato digestivo). FORMA DE EMPLEO: se toma el cocimiento de la flor.

Trigonospermum melampodioides DC. (2) PARTE UTILIZADA: hoja, toda la planta. USO: medicinal (enfermedades del hígado, enfermedades de la mujer). FORMA DE EMPLEO: se toma el cocimiento de las hojas y de la planta completa. 
Verbesina klattii B.L. Rob. \& Greenm. PARTE UTILIZADA: toda la planta. USO: forrajera. FORMA DE EMPLEO: pastoreo cerril (Hernández, 2005).

\section{BEGONIACEAE}

Begonia gracilis HBK. $(7,8)$ "sangre de toro", "flor de agosto", "tsitsiki agosturi", "toruri iurhiri". PARTE UTILIZADA: flor, tallo. USO: melífera, ornato, comestible. FORMA DE EMPLEO: Colecta; el tallo se consume fresco.

\section{BERBERIDACEAE}

Berberis moranensis Hebenstr. \& Ludw. "palo amarillo". PARTE UTILIZADA: fruto. USO: tóxico. FORMA DE EMPLEO: El fruto es consumido fresco (Hernández, 2005).

\section{BETULACEAE}

Alnus jorullensis H.B.K. subsp. lutea Furlow. $(24,28)$ "tupamu". PARTE UTILIZADA: tallo, hoja. USO: maderable (artesanías), medicinal (fiebre, estreñimiento). FORMA DE EMPLEO: madera aserrada; se prepara la infusión de un manojo de hojas en 10 litros de agua y se baña al enfermo hasta que ceda la fiebre; se prepara la infusión de un puño de hojas en 1 litro de agua se toma una taza tres veces al día.

Alnus acuminata ssp. arguta (Schltdl.) Furlow. (24) “aile". PARTE UTILIZADA: tallo. USO: maderable (elaboración de muebles). FORMA DE EMPLEO: madera aserrada.

\section{BORAGINACEAE}

Lasiarrhenum strigosum (H.B.K) Johnst. (3). PARTE UTILIZADA: toda la planta. USO: ornamental. FORMA DE EMPLEO: colecta.

\section{CAMPANULACEAE}

Lobelia fenestralis Cav. PARTE UTILIZADA: toda la planta. USO: ceremonial. FORMA DE EMPLEO: con la parte aérea adornan altares (Hernández, 2005).

Lobelia laxiflora H.B.K. $(9,11)$ "tiriru-tsitsiki", "santa Martha". PARTE UTILIZADA: toda la planta, fruto USO: medicinal (erupciones en la piel), ornato, ceremonial, doméstico. FORMA DE EMPLEO: hervida y untada; ornato; con la parte aérea adornan altares; con el fruto se alimentan aves en cautiverio.

\section{CARYOPHYLLACEAE}

Drymaria villosa Cham. \& Schl. (2, 3, 14, 17) "cilantro-arimbicua", "kulandu", "nube de campo". PARTE UTILIZADA: toda la planta. USO: forrajero, ceremonial. FORMA DE EMPLEO: pastoreo cerril; con la parte aérea adornan altares.

\section{CHENOPODIACEAE}

Chenopodium ambrosioides L. (31) "epazote", "kuatsihinasi". PARTE UTILIZADA: toda la planta. USO: medicinal (anginas, diarrea, parásitos intestinales). FORMA DE EMPLEO: se toma el cocimiento de una taza en medio litro de agua (anginas, parásitos intestinales), en un litro de agua (diarrea), se hacen gárgaras (anginas) y se toma tres veces al día (diarrea) y se toma en ayunas (parásitos intestinales)

\section{CISTACEAE}

Helianthemum glomeratum Lag. (31) "Cenicilla", "xarakata-tsitsiki". PARTE UTILIZADA: flor y hoja. USO: medicinal (empacho, diarrea y tos). FORMA DE EMPLEO: hervida con carbonato, cincollaga (Tagetes lucida) y raíz de lucema (Salvia lavanduloides) y tomada como té. 
Bello González, M.A. et al.: Plantas útiles de la comunidad indígena Nvo. Sn. Juan Parangaricutiro, Mich., Méx.

\section{CLETHRACEAE}

Clethra mexicana A. DC. $(3,31)$ "pácata", "cucharillo". PARTE UTILIZADA: tallo, flores. USO: maderable (artesanal, figuras talladas), melífera. FORMA DE EMPLEO: madera aserrada.

\section{CONVOLVULACEAE}

Cuscuta corymbosa Ruíz \& Pavón. (4, 9, 21) "tripilla", "tiripo", "putsukata tsauapiti”. PARTE UTILIZADA: tallo. USO: medicinal (contra mezquinos), ornamental. FORMA DE EMPLEO: el tallo se aplica localmente con "ruda" y "anis"; adorno sobre árboles de navidad.

\section{CORIARIACEAE}

Coriaria ruscifolia L. (5, 12, 13, 15, 17, 18, 32) "hierba del borrego", "kitzirin", "cola de borrego", "karichi t'ireku", "karichiri ch'eti”. PARTE UTILIZADA: hojas, ramas y fruto. USO: medicinal (pulmonía), doméstico (alimento para aves en cautiverio), tóxica para el hombre (fruto). FORMA DE EMPLEO: se prepara la infusión de una rama de la planta, mezclada con "guayacán” (Sweetia) y "cirian” (Crescentia), se toma una taza tres veces al día; colecta.

\section{CRUCIFERAE}

Brassica campestris L.(12) "mostacilla", "reina". PARTE UTILIZADA: fruto, toda la planta. USO: medicinal (limpieza del intestino e inflamación del hígado) comestible, alimento para aves en cautiverio. FORMA DE EMPLEO: se prepara una infusión con un puñado de la planta (toda la planta) para un litro de agua, se toma una taza tres veces al día; la hoja tierna guisada.

Brassica nigra (L.) Koch. (4, 6, 8, 10, 11, $13,14,15,18)$ "hierba del golpe". PARTE
UTILIZADA: toda la planta, hojas. USO: medicinal (golpes), comestible. FORMA DE EMPLEO: se prepara el cocimiento con un puñado de planta en un litro de agua, se cuela y se aplica con lienzos y/o compresas en la parte afectada; la hoja tierna guisada.

Lepidium virginicum L. (1) "lentejilla". PARTE UTILIZADA: toda la planta, hojas. USO: medicinal (vómito). FORMA DE EMPLEO: se prepara el cocimiento con una taza de planta en un litro de agua, se toma una taza tres veces al día.

Romanschulzia arabiformis (DC.) Rollins. PARTE UTILIZADA: hojas. USO: comestible. FORMA DE EMPLEO: la hoja tierna guisada (Hernández, 2005).

\section{ERICACEAE}

Arbutus xalapensis H.B.K. (3, 12, 14, 15, 16, 17, 19, 20) "madroño", "banuntzin", "panagsi”. PARTE UTILIZADA: tronco, fruto, corteza. USO: maderable (artesanías, fabricación de ensaladeras, dulceros, especieros, floreros, platos, saleros, juguetes, cofres, licoreras), doméstico (alimento para aves en cautiverio) medicinal (astringente en casos de diarrea). FORMA DE EMPLEO: madera aserrada, colecta, infusión y tomada como té.

Arctostaphylos discolor (Hook.) DC. (15, 20, 23) "madroño", "madronillo", "kapemu", "cuentu-checua", "panagsi”. PARTE UTILIZADA: fruto, flor. USO: medicinal (afecciones del riñón), doméstico (alimento para aves en cautiverio). FORMA DE EMPLEO: se prepara la cocción de un puño de la planta (parte aérea) en un litro de agua, se toma como agua de uso; el fruto es tóxico para el consumo humano. 
Pernettya ciliata (Sclecht. \& Cham.) Sma11. PARTE UTILIZADA: toda La planta. USO: ceremonial. FORMA DE EMPLEO: la parte aérea es utilizada en adornos para los altares (Hernández, 2005).

\section{EUPHORBIACEAE}

Euphorbia ocymoidea L. (4, 6). PARTE UTILIZADA: toda la planta. USO: forrajera FORMA DE EMPLEO: pastoreo cerril.

Euphorbia radians var. stormiae (Croizat) Rzed. \& Calderón. (27, 30,) "catarinita". PARTE UTILIZADA: toda la planta. USO: medicinal (afecciones del riñón). FORMA DE EMPLEO: se prepara la cocción de un puño de la planta en un litro de agua, se toma como agua de uso.

\section{FAGACEAE}

Quercus candicans Née. $(10,11)$ "encino de asta", "encino blanco". PARTE UTILIZADA: tronco. USO: maderable (leña, postes para cerca, mangos y cabos para herramienta e implementos agrícolas, horcones, rayos de carreta, redilas para camiones, cajas para empaque), carbón. FORMA DE EMPLEO: madera aserrada (Bello y Labat, 1987).

Quercus castanea Née. $(8,10,12)$ "encino blanco", "encino roble". PARTE UTILIZADA: tronco. USO: maderable (leña, postes para cerca, horcones, arados, cabos para herramienta, ejes para carreta), carbón, artesanía (trompos y baleros). FORMA DE EMPLEO: madera aserrada (Bello y Labat, 1987).

Quercus crassifolia Humb. \& Bonpl. (22, 27) "encino pepitillo", "encino colorado", "encino prieto". PARTE UTILIZADA: tronco. USO: maderable (leña, postes para cerca y plataformas para camiones), carbón. FORMA DE EMPLEO: madera aserrada (Bello y Labat, 1987).

Quercus crassipes Humb. \& Bonpl. (27, 31, 32) "encino pepitillo", "encino colorado". PARTE UTILIZADA: tronco. USO: maderable (leña, postes para cerca, cabos para herramienta y azadones, horcones) (artesanías (trompos y baleros), carbón, medicinal (afianzar la dentadura). FORMA DE EMPLEO: madera aserrada; con el cocimiento de la corteza se enjuaga la boca (Bello y Labat, 1987).

Quercus dysophylla Benth. (3,10). PARTE UTILIZADA: tronco. USO: maderable (leña, postes para cerca). FORMA DE EMPLEO: madera aserrada (Bello y Labat, 1987).

Quercus laurina Humb. \& Bonpl. (6, 7, 22, 25, 26, 28, 30, 32) "encino laurelillo", “encino prieto". PARTE UTILIZADA: tronco. USO: maderable (leña, postes para cerca, mangos y cabos para herramienta e implementos agrícolas, redilas para camiones), artesanías (vaquetas para tambor), carbón, comestible, medicinal (afianzar la dentadura). FORMADE EMPLEO: madera aserrada; para la elaboración de tortillas, el fruto del encino es pelado, cocido y mezclado con salvado; con el cocimiento de la corteza se enjuagan la boca (Bello y Labat, 1987).

Quercus martinezii C.H. Muller. (11) “encino colorado", “encino prieto". PARTE UTI-

LIZADA: tronco. USO: maderable (leña, postes para cerca, cabos para herramienta y horcones). FORMA DE EMPLEO: madera aserrada (Bello y Labat, 1987). 
Bello González, M.A. et al.: Plantas útiles de la comunidad indígena Nvo. Sn. Juan Parangaricutiro, Mich., Méx.

Quercus obtusata Humb.\& Bonpl. (2, 6, 7, 13) "encino blanco", "encino prieto", "encino roble". PARTE UTILIZADA: tronco. USO: maderable (leña, postes para cerca, implementos agrícolas (arados), horcones, cabos para herramienta), carbón, curtir pieles, medicinal (afianzar la dentadura). FORMA DE EMPLEO: madera aserrada; con el cocimiento de la corteza se enjuagan la boca (Bello y Labat, 1987).

Quercus rugosa Née. (3, 26, 27, 30, 31, 32) "encino roble", "encino prieto". PARTE UTILIZADA: tronco. USO: maderable (leña, postes para cerca, cabos para herramienta); carbón, comestible (Se elabora con la bellota café y tortillas), medicinal (para afianzar la dentadura). FORMA DE EMPLEO: madera aserrada; para la elaboración de café el fruto del encino es pelado y cocido; para la elaboración de la tortilla el fruto del encino es pelado, cocido y mezclado con salvado; con el cocimiento de la corteza se enjuagan la boca (Bello y Labat, 1987).

\section{GARRYACEAE}

Garrya laurifolia Hartw. (21, 28, 30) "azulillo". PARTE UTILIZADA: tronco, corteza y hojas. USO: maderable (artesanías; figuras talladas); medicinal (diarrea crónica). FORMA DE EMPLEO: madera aserrada; extracto con las hojas y tomar en gotas.

\section{GERANIACEAE}

Erodium cicutarium (L) L'Hérit. $(1,6)$ "alfilerillo", "pikuku jasi". PARTE UTILIZADA: toda la planta. USO: forrajera. FORMA DE EMPLEO: pastoreo cerril.

Geranium aristisepalum Moore. (1, 2, 14, 19) "fresa-Misco", "puesi tireku", "akuku". PARTE UTILIZADA: toda la planta. USO: forrajera. FORMA DE EMPLEO: pastoreo cerril.

Geranium latum Small. (3, 6, 7, 9, 11, 17, 20, 21, 22, 23, 24, 28, 30) "chipihuite", "pachihuite", "pata de león". PARTE UTILIZADA: raíz, toda la planta. USO: medicinal (enfermedades del aparato digestivo, dolor de muelas, rozaduras de bebés). FORMA DE EMPLEO: la raíz machacada es aplicada localmente.

Geranium seemannii Peyr. (1, 4, 5, 11, 13, 15, 20, 22, 23, 25) "pata de León", "palmita", "juria-terango", "paralaja-kula", "pukiri jantsiri". PARTE UTILIZADA: toda la planta. USO: medicinal (contra rozaduras, llagas, golpes, granos, diarrea, cólicos), forrajera. FORMA DE EMPLEO: molida y untada; hervida y lavados; hervida y tomada como té; pastoreo cerril.

\section{LAMIACEAE}

Hypsis mutabilis (Rich.) Briq. $(5,13,16)$ "chía". PARTE UTILIZADA: toda la planta. USO: medicinal (diarrea), ceremonial. PARTE UTILIZADA: raíz, toda la planta. FORMA DE EMPLEO: se prepara el cocimiento de un puño de raíz de chía en $3 / 4$ de litro, se toma una taza tres veces al día hasta que ceda la diarrea; la parte aérea es utilizada en adornos para altares.

Lepechinia caulescens (Ort.) Epling.(4, $8,17,18,27)$ "chichinatztacua", "salvia". PARTE UTILIZADA: hoja, toda la planta. USO: medicinal (gastritis, hinchazón y aire en los oídos, bilis, "torzón" de frío). FORMA DE EMPLEO: hervida y tomada como té; colocada en forma de "tapones" en lo oídos; hervida con canela, piloncillo y tomada como té. 
Salvia elegans Vahl. $(1,6,9,15,18,22$, 23, 24, 25) "tiri-tsitsiki", "huataranapu", "limoncillo", "flor del cerro". PARTE UTILIZADA: toda la planta. USO: medicinal (contra el vómito), ceremonial, ornamental. FORMA DE EMPLEO: hervida con canela y tomada como té; se utiliza en "limpias"; decoración de iglesias en las bodas.

Salvia gesneriflora Lindl. (1, 2, 4, 14) "flor de Tzintzungaraman", "flor de colibrí". PARTE UTILIZADA: flor. USO: ceremonial. FORMA DE EMPLEO: se hacen coronas para los santos (Hernández, 2005).

Salvia lavanduloides Kunth. (1, 13, 16, 17, 19, 23, 24) "lucema", "mazorquilla", "alucema", "aguanda-tsitsiki", "chía cimarrona", "flor del cielo", "k'uironi simarroni". PARTE UTILIZADA: toda la planta, flor. USO: medicinal (diarrea, vómito y bilis, enfermedades ginecológicas y parálisis), ceremonial, melífera. FORMA DE EMPLEO: hervida con canela y tomada como té ( 3 o 4 cucharadas antes de cada comida); hervida con el tizne de cazo y tomada como agua de uso; se hacen "limpias" o baños.

Salvia mexicana var. minor Benth. (1, 4, 5, $6,8,9,11,12,13,15,16,17,19,20,21$, 22, 24) "chía", "charahuesca", "ichukuta", "azul-sipari”. PARTE UTILIZADA: hoja, semilla, flor, toda la planta. USO: medicinal (anticalculosa en riñones, aparato digestivo y respiratorio) doméstico, comestible, melífera, forrajera. FORMA DE EMPLEO: hervida y tomada como agua de uso; las hojas son utilizadas como estropajo; la semilla molida sirve para hacer agua fresca; pastoreo cerril.

Salvia mocinoi Benth. PARTE UTILIZADA: toda la planta. USO: ceremonial.
FORMA DE EMPLEO: la parte aérea es utilizada en adornos para los altares (Hernández, 2005).

Salvia polystachya Ort. $(6,7,18)$ "lucemilla", "azulema" "chía". PARTE UTILIZADA: semilla. USO: medicinal (dolor de estómago, enfermedades de la mujer), comestible. FORMA DE EMPLEO: se toma el cocimiento de la parte aérea mezclada con "lantén" (Plantago australis); con la semilla molida se prepara agua fresca.

Salvia purpurea Cav. (4, 11, 12, 15, 23, 24) "flor de morada", "ramoncillo", "sirani tsitsiki". PARTE UTILIZADA: toda la planta; flor. USO: medicinal (dolor de estómago), ceremonial, ornamental. FORMA DE EMPLEO: se toma el cocimiento de un puño de la parte aérea de la planta mezclada con lantén (Plantago australis) en medio litro de agua, se toma en el momento del dolor; se hacen coronas para los santos; adornos florales.

Salvia thyrsiflora Benth. PARTE UTILIZADA: toda la planta. USO: ceremonial. FORMA DE EMPLEO: la parte aérea es utilizada para adornar los altares durante las festividades religiosas (Hernández, 2005).

Satureja macrostema (Benth.). (6, 9, 15, 20, 25) "nurite" "nurhitini te". PARTE UTILIZADA: toda la planta. USO: medicinal (dolor de estómago y tos). FORMA DE EMPLEO: hervida y tomada como té.

\section{LAURACEAE}

Persea americana Mill. (31) "aguacate criollo”. PARTE UTILIZADA: hoja. USO: medicinal (antiséptico). FORMA DE EMPLEO: se prepara el cocimiento de una taza 
Bello González, M.A. et al.: Plantas útiles de la comunidad indígena Nvo. Sn. Juan Parangaricutiro, Mich., Méx.

de hojas de aguacate en $3 / 4$ de litro de agua y se lava la cortada con este cocimiento.

\section{LEGUMINOSAE}

Acacia angustissima (Mill.) Kuntze. (17, 19) "timbique", "timbin". PARTE UTILIZADA: raíz. USO: medicinal (dolor de garganta, afianzar la dentadura), ceremonial, forrajera. FORMADE EMPLEO: se utiliza el cocimiento de la raíz, se hacen gárgaras; se mastica la corteza (afianzar); la parte aérea es emplea en adornos para los altares durante las festividades religiosas.

Astragalus lyonnetii Barneby. PARTE UTILIZADA: toda la planta. USO: ceremonial. forrajera. FORMA DE EMPLEO: la parte aérea es empleada en adornos para los altares durante las festividades religiosas (Hernández, 2005).

Calliandra grandiflora (L'Hér) Benth. $(5,13)$ "cachachakua", "pelo de ángel". PARTE UTILIZADA: hoja. USO: medicinal (tos ferina), forrajera. FORMA DE EMPLEO: se prepara el cocimiento de un pedacito de rama de la planta en medio litro de agua, se toma una taza endulzada con miel de abeja en ayunas y otra al acostarse; pastoreo cerril.

Cassia laevigata Willd. "flor de sinverguenza". PARTE UTILIZADA: toda la planta. USO: ceremonial. FORMA DE EMPLEO: con la parte aérea se hacen "limpias" (Hernández, 2005).

Cassia tomentosa L. "flor de sinverguenza". PARTE UTILIZADA: toda la planta. USO: ceremonial, ornamental, tóxico. FORMA DE EMPLEO: con la parte aérea de la planta se adornan los altares durante las festividades religiosas; la planta es plan- tada en los patio; el fruto ingerido es tóxico para el ser humano (Hernández, 2005).

Crotalaria longirostrata Hook. \& Arn. $(5,9)$ "sonajilla", "vaca", "empanadilla", "kurhinditu”. PARTE UTILIZADA: toda la planta, flor. USO: medicinal (antidiarréico en niños), comestible, melífera. FORMA DE EMPLEO: hervida y tomada como té; la flor es guisada con cilantro y ajo.

Crotalaria rotundifolia (Walter) J.F. Gmel. $(8,13,16)$ "sonajilla", "chechinastakua". PARTE UTILIZADA: toda la planta, flor. USO: medicinal (anticoagulante, cólicos). FORMA DE EMPLEO: se prepara un cocimiento de la planta en un litro de agua, se aplica en forma de compresas; se toma una taza en el momento del cólico.

Crotalaria pumila Ort. (3) "toru-tsitsiki", "flor de toro". PARTE UTILIZADA: toda la planta. USO: ornamental. FORMA DE EMPLEO: en macetas y floreros (Hernández, 2005).

Dalea brachystachya Gray. "karatacua”, "escoba". PARTE UTILIZADA: hoja, rama. USO: doméstico. FORMA DE EMPLEO: se hacen escobas (Hernández, 2005).

Dalea elata Hook. \& Arn. "rudilla". PARTE UTILIZADA: toda la planta. USO: ceremonial. FORMA DE EMPLEO: con la parte aérea de la planta se adornan los altares (Hernández, 2005).

Dalea obovatifolia Ort. PARTE UTILIZADA: toda la planta. USO: ornamental. FORMA DE EMPLEO: con la parte aérea de la planta se adornan floreros (Hernández, 2005). 
Dalea versicolor Zucc.(4) "hierba del zorrillo", "k'uitsiku xukurhi". PARTE UTILIZADA: toda la planta. USO: medicinal (anticoagulante). FORMA DE EMPLEO: hervidas y como cataplasmas.

Desmodium subsessile Schl. (6, 8, 10, 11, $12,13,14)$ "amor seco" "hierba del arlomo". PARTE UTILIZADA: toda la planta. USO: medicinal (piquete de insecto y/o larva "arlomo", padecimientos de la piel), forrajera. FORMA DE EMPLEO: una vez cocida la parte aérea de la planta se aplican fomentos en la parte afectada

Crotalaria pumila Ort. PARTE UTILIZADA: toda la planta. USO: ceremonial. FORMA DE EMPLEO: con la parte aérea de la planta se adornan los altares (Hernández, 2005).

Lupinus bilineatus Benth. (3, 6) "tzirangueramani", "tziran-tsitsiki", "tamasuchil", "siuatsi". PARTE UTILIZADA: toda la planta. USO: medicinal (antibembericua), fertilizante, ornamental, ceremonial, forrajera. FORMA DE EMPLEO: se prepara el cocimiento de dos tazas de la planta (parte aérea) en dos litros de agua, se baña al enfermo; en las parcelas de cultivo la cortan y dejan que se reincorpore al suelo; en floreros; en las coronas del día de muertos; pastoreo cerril.

Lupinus campestris Cham. \& Schl. (4, 8, $11,14,15,16,20,21,22,23,23,24,25,26$, 27, 28, 29) "tamazuchil", "siuatsi". PARTE UTILIZADA: toda la planta, flor. USO: medicinal (herpes, antibembericua, dolor de muelas, diarrea, vómito y empacho de niños), fertilizante (abono verde), forrajera, ceremonial, ornato. FORMA DE EMPLEO: los brotes tiernos molidos se aplican direc- tamente en la parte afectada; se prepara el cocimiento de un pedazo de raíz en medio litro de agua se toma una taza tres veces al día hasta que ceda el vómito, la diarrea y el empacho; se incinera y se aplica al suelo; pastoreo cerril; con las flores se adornan altares y coronas el día de muertos; con el cocimiento de la planta completa (brotes tiernos) se aplican cataplasmas; con la planta completa se adornan floreros.

Mimosa albida H. \& B. (5) "sinverguenza", "k'uiratsiku noukari". PARTE UTILIZADA: raíz. USO: veterinario (parásitos intestinales en caballos y burros). FORMA DE EMPLEO: hervida y tomada.

Phaseolus coccineus L. (4) "frijolillo", "tatsin-chucuri". PARTE UTILIZADA: toda la planta. USO: forrajera. FORMA DE EMPLEO: pastoreo cerril.

Phaseolus heterophyllus Willd. (4,7) "frijolillo", "tatsini sapichu". PARTE UTILIZADA: toda la planta. USO: forrajera. FORMA DE EMPLEO: pastoreo cerril.

Phaseolus vulgaris L. "sonajilla". PARTE UTILIZADA: fruto, raíz. USO: doméstico. FORMA DE EMPLEO: el fruto se utiliza como alimento para aves en cautiverio e ingerida la raíz por el ser humano es tóxica (Hernández, 2005).

Trifolium amabile HBK. (4, 5, 6, 4, 8, 9, 11, $12,13,15,16,17,20,19)$ "trébol”, “carretilla", "pitangua", "uirhijpiku sapichu”. PARTE UTILIZADA: toda la planta, flor. USO: medicinal (fuego labial), forrajera, melífera, medicinal (postemillas). FORMA DE EMPLEO: se prepara el cocimiento de una taza de la planta (parte aérea) en un litro de agua, se hacen enjuagues bucales 
Bello González, M.A. et al.: Plantas útiles de la comunidad indígena Nvo. Sn. Juan Parangaricutiro, Mich., Méx.

(buches); pastoreo cerril; con el cocimiento de la planta completa se hacen enguagues bucales (buches).

Vicia humilis H.B.K. $(1,2,4)$. PARTE UTILIZADA: toda la planta. USO: forrajera. FORMA DE EMPLEO: pastoreo cerril.

Zornia thymifolia HBK. (5, 13, 16) "hierba de la víbora". PARTE UTILIZADA: toda la planta. USO: medicinal (diarrea, intoxicación, purificar la sangre). FORMA DE EMPLEO: se prepara el cocimiento de un puño de la planta en un litro de agua, se toma una taza en ayunas tres veces al día durante nueve días.

\section{LOGANIACEAE}

Buddleia cordata HBK $(6,27)$ "tepozan", "tepoja". PARTE UTILIZADA: tronco, corteza y raíz. USO: maderable aserrada (Artesanías; elaboración de collares y figuras talladas); medicinal (diurético, afecciones uterinas, digestivas y reumáticas). FORMA DE EMPLEO: madera aserrada; hervida la corteza y la raíz y tomada como agua de uso.

Buddleia sessiliflora H.B.K.(1, 30) "tepuza", "pupurachku”. PARTE UTILIZADA: hoja. USO: medicinal (postemillas). FORMA DE EMPLEO: hervida con carbonato y aplicada directamente en la postemilla.

\section{LORANTHACEAE}

Arceuthobium globosum Hawksworth \& Wiens. "injerto", “muérdago". PARTE UTILIZADA: toda la planta USO: ceremonial. FORMA DE EMPLEO: la parte aérea es utilizada para adornar altares durante las festividades religiosas (Hernández, 2005).

\section{LYTHRACEAE}

Cuphea aequipetala Cav. (12) "hierba del cáncer", "calavera", "uarhiku”. PARTE UTILIZADA: toda la planta. USO: medicinal (heridas y tumores), ritual. FORMA DE EMPLEO: con la cocción de planta se lava la parte afectada o se aplica en forma de cataplasmas; hervida con sal y tomada como té.

Cuphea jorullensis H.B.K. (12) "manga de niño", "jaskurhaku sapiri". PARTE UTILIZADA: flor. USO: medicinal (tos y bronquitis). FORMA DE EMPLEO: hervida y tomada como té.

Cuphea wrightii A. Gray. (5) "calaver-tsitsiki”. PARTE UTILIZADA: toda la planta. USO: medicinal (empacho). FORMA DE EMPLEO: con el cocimiento de una taza de planta (parte aérea) en un litro de agua, se toma una taza en ayunas y otra al acostarse.

\section{MALVACEAE}

Kearnemalvastrum lacteum (Ait) Bates. (9, $15,17)$ "malvavisco", "malba niregarhi" "huinare blanco". PARTE UTILIZADA: toda la planta, raíz. USO: medicinal (fiebre, dolor e inflamación del estómago), forrajera, comestible FORMA DE EMPLEO: se prepara un cocimiento de un puño de la planta (hojas) en medio litro de agua, se toma una taza tres veces al día y las hojas molidas se aplican directamente en la parte inflamada; pastoreo cerril.

Sida rhombifolia L. (1, 5, 11, 12, 13, 16, 17, 19, 20, 21) "huinare". PARTE UTILIZADA: toda la planta, hoja. USO: medicinal (tifoidea, dolor de cabeza, llagas, granos, golpes, dolor de estómago), forrajera. 
FORMA DE EMPLEO: las hojas de la planta se muelen con carbón de "ocote", se cuela y se toma (tifoidea); las hojas se muelen con sal y se aplican directamente en llagas, granos y golpes; se prepara el cocimiento de un puño de la planta en medio litro de agua, se toma una taza tres veces al día hasta que ceda el dolor de estómago; pastoreo cerril.

\section{ONAGRACEAE}

Epilobium ciliatum Raf. PARTE UTILIZADA: toda La planta. USO: ceremonial. FORMA DE EMPLEO: la parte aérea es utilizada en adornos para altares durante las festividades religiosas (Hernández, 2005).

Fuchsia fulgens D.C. "gallito-Tiringuin", "cempoasuchili". PARTE UTILIZADA: fruto. USO: comestible. FORMA DE EMPLEO: colecta (Hernández, 2005).

Fuchsia microphylla H.B.K. (1,4,5,6,18) "kupanda-arimbicua", "aguacate". PARTE UTILIZADA: fruto, flor. USO: tóxico; ornamental

Fuchsia thymifolia H.B.K. (1, 4, 5) "azulema", "uarhoti piriraku". PARTE UTILIZADA: flor, toda la planta. USO: ornamental, melífera. FORMA DE EMPLEO: adornos florales.

Lopezia racemosa Cav. (1, 6, 4, 8, 9, 11, 12, $13,14,15,16,17,20,21,22,23,27,28)$ "enandimisu", "guayabilla", "alfilerillo", "peritas". PARTE UTILIZADA: toda la planta, raíz. USO: medicinal (contra granos, anginas y paperas, tos, abortiva), forrajera. FORMA DE EMPLEO: con la cocción de la planta se lava el grano o mezclado con carbonato se hacen gárgaras; hervida $\mathrm{y}$ tomada como té; pastoreo cerril.
Oenothera purpusii Munz. "tempranera". PARTE UTILIZADA: toda la planta. USO: ceremonial. FORMA DE EMPLEO: la parte aérea es utilizada en adornos para altares durante las festividades religiosas (Hernández, 2005).

Oenothera rosea L'Hér ex Aiton. (17) "hierba del golpe". PARTE UTILIZADA: toda la planta. USO: medicinal (golpes) FORMA DE EMPLEO: se prepara un cocimiento de un manojo de la planta (100 g) en un litro de agua o se macera la misma cantidad en un litro de alcohol, se aplica en forma de fomentos calientes en la parte afectada o untada la tintura después de 15 días de maceración.

\section{OXALIDACEAE}

Oxalis corniculata L. (7) "trébol del campo". PARTE UTILIZADA: toda la planta. USO: forrajera. FORMA DE EMPLEO: pastoreo cerril.

Oxalis hernandesii DC. $(5,8,16)$ "kaguima-arimbicua”. PARTE UTILIZADA: flor. USO: comestible. FORMA DE EMPLEO: guisada.

\section{PHYTOLACCACEAE}

Phytolacca icosandra L. (1, 4, 12, 16) "conguera-man", "conguera", "konguerani", “elote jabonoso". PARTE UTILIZADA: hoja, fruto y raíz. USO: comestible, doméstico, medicinal (piquete de insecto, llagas, granos). FORMA DE EMPLEO: la hoja tierna guisada; del fruto y raíz se extrae la jabonadura para el pelo y la ropa; se aplica la tintura elaborada del fruto para cataplasmas o se prepara el cocimiento de una taza de la planta en un litro de agua y se aplica directamente en la llaga. 
Bello González, M.A. et al.: Plantas útiles de la comunidad indígena Nvo. Sn. Juan Parangaricutiro, Mich., Méx.

\section{PLANTAGINACEAE}

Plantago australis Lam. (4, 6, 9, 11, 12, 14, $15,17,19,20,24)$ "lantén", "ylechichucali", "yantén". PARTE UTILIZADA: toda la planta. USO: medicinal (diarrea, empacho, torzón, tos). FORMA DE EMPLEO: hervida con "manzanilla", canela, hojas de guayaba y tomada como té.

\section{POLEMONIACEAE}

Loeselia mexicana (Lam.) Brand. (2, 16, 19) "espinosilla", "checamicua tsitsiki", "espinosa", "tsitsiki tsurhumu". PARTE UTILIZADA: hoja, toda la planta. USO: medicinal (diarrea, "mal de orina", várices, tos, calvicie), ritual, doméstico. FORMA DE EMPLEO: hervida y tomada como agua de uso; con la cocción de la hoja se aplica con lienzos calientes en la parte afectada; se hacen "limpias"; estrujada toda la planta se obtiene una laca utilizada para el peinado.

\section{POLYGALACEAE}

Monnina schlechtendaliana D. Dietr. (1, 5, 6, 12, 13, 15, 17, 20, 19, 27) "aguacatillo". PARTE UTILIZADA: fruto, toda la planta. USO: medicinal (deshidratación infantil), doméstico. FORMA DE EMPLEO: se toma el cocimiento de un puño de la planta en un litro de agua, se toma como agua de uso; el fruto es usado como alimentos para aves en cautiverio; el fruto es tóxico para el ser humano.

\section{POLYGONACEAE}

Rumex crispus L. "lengua de vaca", "vara colorada", "quelite". PARTE UTILIZADA: hoja. USO: comestible. FORMA DE EMPLEO: La hoja se prepara en mole (Hernández, 2005).

\section{RANUNCULACEAE}

Clematis dioica L. (9, 11, 14, 15, 17, 20). PARTE UTILIZADA: toda la planta. USO: medicinal (padecimientos de la piel), ornamental. FORMA DE EMPLEO: el cocimiento de la parte aérea se aplica en fomentos; la parte aérea se pinta y se emplea como naturaleza muerta.

Ranunculus petiolaris HBK. $(8,16)$ "picosilla", "p'ameri xukurhi”. PARTE UTILIZADA: toda la planta. USO: forrajera. FORMA DE EMPLEO: pastoreo cerril.

\section{RHAMNACEAE}

Ceanothus coeruleus Lag. (15) "chavin", "caninda-balis", "palo colorado", "vara colorada". PARTE UTILIZADA: toda la planta, fruto. USO: medicinal (golpes), doméstico, forrajera. FORMA DE EMPLEO: en un litro de agua se coloca media taza de la planta en cocimiento, se aplica en compresas; el fruto es usado como alimento para aves en cautiverio; pastoreo cerril.

\section{ROSACEAE}

Acaena elongata L. (3, 4, 7, 8, 9, 11, 15, 18, 22, 24, 25, 26, 28, 29) "kuchacheka", "cadillo", "xukurhi uakasi tirekua". PARTE UTILIZADA: raíz, toda la planta. USO: medicinal (afecciones del riñón, reumatismo), forrajera. FORMA DE EMPLEO: se prepara un cocimiento de un puño de raíz de la planta en un litro de agua, se toma como agua de uso o se aplica localmente en caso de dolor reumático; pastoreo cerril.

Alchemilla procumbens Rose. (9, 11, 15, 18, 24) "pata de león”, "puki jatsiri”. PARTE UTILIZADA: toda la planta. USO. medicinal (anticoagulante y antiinflamatoria), forrajera. FORMA DE EMPLEO: hervida y aplicada en cataplasmas; pastoreo cerril. 
Crataegus pubescens (H.B.K.) Steud. (3, 22, 24, 27, 29) "tejocote", "manzanilla", "karhasi". PARTE UTILIZADA: fruto, hoja. USO: medicinal (tos, diurético); comestible, forrajera. FORMA DE EMPLEO: se prepara un cocimiento de una taza de flor y fruto de la planta en un litro de agua, se toma una taza tres veces al día hasta que ceda la tos, se prepara un cocimiento de 100 g de raíz de la planta en dos litros de agua, se toma una taza en ayunas y como agua de uso; colecta; pastoreo cerril.

Prunus serotina Ehrh. "capulin". PARTE UTILIZADA: fruto. USO: comestible. FORMA DE EMPLEO: el fruto es consumido en fresco (Hernández, 2005).

Rubus caudatisepalus Calderón. $(6,15)$ "zarzamora". PARTE UTILIZADA: fruto. USO: comestible. FORMA DE EMPLEO: consumo directo o se elaboran mermeladas $\mathrm{y}$ atole.

Rubus liebmannii Focke. (17, 22, 30) "zitun". PARTE UTILIZADA: fruto, raíz. USO: comestible, medicinal (afecciones digestivas, molestias al brote de la dentición de bebés). FORMA DE EMPLEO: consumo directo; se toma el cocimiento de la raíz.

Rubus pringlei Rydb. $(3,19)$ "zarzamora". PARTE UTILIZADA: fruto. USO: medicinal (diarrea), comestible. FORMA DE EMPLEO: se prepara el cocimiento de un puño de la planta en un litro de agua, se toma una taza tres veces al día; consumo directo o se elaboran mermeladas y atole.

\section{RUBIACEAE}

Bouvardia ternifolia (Cav.) Schlecht. (5, $30,31)$ "hierba de la vibora", "cigarrillo", akuitsi uarhiraku". PARTE UTILIZADA: toda la planta. USO: medicinal (prurito, afecciones urinarias), ornamental, ritual. FORMA DE EMPLEO: hervida y tomada como té; arreglos florales (colecta) y adorno de templos.

Crusea diversifolia (HBK.) Anderson. $(4,8)$. PARTE UTILIZADA: toda la planta. USO: forrajera. FORMA DE EMPLEO: pastoreo cerril.

\section{SCROPHULARIACEAE}

Castilleja arvensis Cham. \& Schlecht. $(22,24,25,26,28)$ "flor de Angélica" "sagalejilla". PARTE UTILIZADA: toda la planta. USO: medicinal (afecciones del riñón), ceremonial, ornamental. FORMA DE EMPLEO: se prepara un cocimiento de una taza de flores de la planta en un litro de agua, se toma una taza tres veces al día; con la parte aérea se adornan los altares durante las festividades religiosas; la parte aérea es colocada en floreros.

Castilleja gracilis Benth. (5, 13, 14, 16, 19, 20). PARTE UTILIZADA: toda la planta. USO: medicinal (afecciones del aparato respiratorio y digestivo), ceremonial. FORMA DE EMPLEO: se toma el cocimiento de la parte aérea mezclado con flor de "mirasol" y se toma una taza tres veces al día hasta que ceda la tos; se toma el cocimiento de la parte aérea mezclada con "cenicilla" (Helianthemum glomeratum), se toma una taza tres veces al día hasta que ceda la diarrea y el empacho; con la parte aérea se adornan las coronas del día de muertos.

Castilleja tenuiflora Benth. (13) "chacamekua”, "espinoso". PARTE UTILIZADA: toda la planta. USO: medicinal (disentería), 
Bello González, M.A. et al.: Plantas útiles de la comunidad indígena Nvo. Sn. Juan Parangaricutiro, Mich., Méx.

ornamental. FORMA DE EMPLEO: hervida y tomada como té; adornos florales.

Lamourouxia multifida H.B.K. (13) "tirindin-tsitsiki", "flor de San Miguel", "flor de arete". PARTE UTILIZADA: flor, toda la planta. USO: melífera, ornamental, forrajera, ritual. FORMA DE EMPLEO: adornos; pastoreo cerril; se utiliza en "limpias".

Lamourouxia xalapensis HBK. PARTE UTILIZADA: toda la planta. USO: ceremonial. FORMA DE EMPLEO: la parte aérea se utilizada en adornos para los altares durante las festividades religiosas (Hernández, 2005).

\section{SOLANACEAE}

Cestrum anagyris Dunal. (12, 20). "huele de noche". PARTE UTILIZADA: toda la planta. USO: ceremonial, doméstico, medicinal (fiebre) FORMA DE EMPLEO: la parte aérea se utilizada en "limpias"; el fruto es utilizado como alimento para aves en cautiverio; con el cocimiento de la parte aérea se baña al enfermo para bajar la fiebre.

Cestrum nitidum Mart. \& Gal. (17) "hediondilla", "xukurhi sipieti". PARTE UTILIZADA: hoja. USO: medicinal (para los niños “chipiles”). FORMA DE EMPLEO: la hoja restregada en el cuerpo del niño.

Cestrum thyrsoideum H.B.K. (1, 4, 5, 6, 7, $8,9,12,13,14,15,16,17,19,20,21,22$, 24, 28, 29, 30) "hediondilla", "tzipiguati", "tzirpiazi”, "tzipiandikua”. PARTE UTILIZADA: fruto, toda la planta, hoja. USO: medicinal (cefaleas, dolor de pulmón, fiebre); comestible (alimento para la fauna silvestre), ritual. FORMA DE EMPLEO: la hoja macerada se aplica en las sienes; la hoja restregada en el pulmón; con la cocción de la planta se baña al enfermo para disminuir la fiebre; toda la planta utilizada para hacer "limpias".

Physalis pringlei Greenm. (1, 2, 6, 7, 15, $18,21,22,23,24,26,27,28,29,30,32)$ "tomate cimarrón", "tomatillo", "jitomati1lo", "xapindicua". PARTE UTILIZADA: fruto, raíz. USO: medicinal (enfermedades del aparto digestivo, enfermedades del hígado), comestible. FORMA DE EMPLEO: se toma el cocimiento de la raíz mezclado con aceite de olivo y chocolate, se toma una taza tres veces al día; se toma el cocimiento de la raíz mezclado con canela, se toma una taza tres veces al día; con el fruto guisado se prepara una salsa.

Physalis stapelioides (Regel) Bitter.(1) "tomate", "toma kuaraki". PARTE UTILIZADA: fruto. USO: comestible. FORMA DE EMPLEO: guisado.

Solanum cervantesii Lag. (1, 11, 13, 16, 28, 31) "pichecua", "pitseku", "hierba mora". PARTE UTILIZADA: fruto. USO: medicinal (enfermedades del hígado, circulatorio, digestivo y padecimientos de la piel), comestible. FORMA DE EMPLEO: se toma el cocimiento de la parte aérea en un litro de agua, se toma como agua de uso; con el cocimiento de la parte aérea se baña al enfermo; colecta para consumo humano

Solanum lanceolatum Cav. (1, 2, 5, 12, 13, 19) "sosa". PARTE UTILIZADA: flor, fruto. USO: medicinal (congestión, sofocación, destete, reumatismo, granos y mezquinos), melífera. FORMA DE EMPLEO: hervida la flor con un antiácido y tomada; el fruto restregado en el seno y 
en la parte con dolor reumático, mezquinos o granos.

Solanum nigrescens Mart. \& Gal. (1, 4, 7, 14) "hierba mora", "chicua", "sirani". PARTE UTILIZADA: toda la planta, fruto. USO: medicinal (destete, catarro constipado). FORMA DE EMPLEO: con la cocción de las planta se baña al bebé; tres gotas del fruto en cada orificio nasal.

\section{STYRACACEAE}

Styrax ramirezii Greenm. (12) "chilacuate". PARTE UTILIZADA: tronco. USO: maderable (artesanías; elaboración de collares y figuras talladas) FORMA DE EMPLEO: madera aserrada sin labrar.

\section{SYMPLOCACEAE}

Symplocos prionophylla Hemsl. (12) "palo blanco". PARTE UTILIZADA: tronco. USO: maderable (artesanías; elaboración de collares y figuras talladas) FORMA DE EMPLEO: madera aserrada sin labrar.

\section{THEACEAE}

Ternstroemia pringlei Standley. $(3,6,11,12)$ "cucharillo", "flor de tila". PARTE UTILIZADA: flor y tronco. USO: medicinal (para los nervios alterados y el sistema nervioso, contra el insomnio); artesanías (elaboración de figuras talladas). FORMA DE EMPLEO: hervida y tomada como té en ayunas y antes de acostarse; madera aserrada.

\section{TILIACEAE}

Tilia mexicana Schlecht. (12) "sirimo", "tilia", "tsirimu tsipata". PARTE UTILIZADA: flor y tronco. USO: medicinal (contra los nervios); artesanías (elaboración de cucharas, figuras talladas). FORMA DE EMPLEO: hervida y tomada como té; madera aserrada.

\section{UMBELLIFERAE}

Daucus montanus H.\& B. (5, 6, 9, 12, 17) "checamikua-espinita", "cilandrillo", "espinoso". PARTE UTILIZADA: toda la planta. USO: medicinal (cólicos en los niños). FORMA DE EMPLEO: hervida y tomada como té o se baña al niño.

Eryngium carlinae Delar. $(9,13)$ "hierba del sapo", "tiamo-kuatr", "motita de sapo", "kokixukurhi”. PARTE UTILIZADA: toda la planta, raíz. USO: medicinal (enfermedades venéreas, "mal de orina", "empacho de agua", fiebre, prurito, próstata), veterinario (sofocón). FORMA DE EMPLEO: con el cocimiento de la planta se baña al enfermo o se toma como agua de uso; mezclada con Buddleia y tomada como té; hervida la raíz con sal y manzanilla y tomada.

Eryngium columnare Hemsl. (6, 8, 15, 17, 19, 20, 22) "hierba del sapo". PARTE UTILIZADA: toda la planta, raíz. USO: medicinal (enfermedades del aparato digestivo, respiratorio, urinario, circulatorio y padecimientos de la piel). FORMA DE EMPLEO: con el cocimiento de la planta y tomada como agua de uso; hervida la raíz con sal y manzanilla y tomada.

\section{VALERIANACEAE}

Valeriana clematis HBK. PARTE UTILIZADA: toda la planta. USO: ceremonial. FORMA DE EMPLEO: la parte aérea es utilizada en adornos de altares durante las festividades religiosas (Hernández, 2005).

Valeriana urticifolia H.B.K. "chacuaarimbicua", "kelite arimbikua". PARTE UTILIZADA: toda la planta. USO: forrajera, ceremonial. FORMA DE EMPLEO: pastoreo cerril; la parte aérea es utilizada 
Bello González, M.A. et al.: Plantas útiles de la comunidad indígena Nvo. Sn. Juan Parangaricutiro, Mich., Méx.

para adornar altares durante las festividades religiosas (Hernández, 2005).

\section{VERBENACEAE}

Verbena bipinnatifida Nutt. $(11,13)$ "moradilla", "alfombrilla", "tatsundiku moradu". PARTE UTILIZADA: toda la planta, flor. USO: medicinal (tos ferina, alergias, ronchas, sordera y diarrea), ritual (susto en niños). FORMA DE EMPLEO: hervida y tomada como agua de uso; restregada en la piel; se calienta la hoja en un comal y se colocan "tapones" en los oídos; hervida y tomada como té; se utiliza en "limpias".

Verbena carolina L. (4, 6, 12, 13, 17, 19) "verbena", "notembucha", "San Juan", "no se casa”. PARTE UTILIZADA: raíz, hoja. USO: medicinal (antibilioso, sordera, gol- pes y heridas, fiebre y calvicie). FORMA DE EMPLEO: hervida y tomada como té; estrujada la hoja, se coloca en forma de "tapones" en los oídos; molida la raíz con sal y se aplica en golpes y heridas; hervida y bañar al enfermo; hervida y aplicada en el cuero cabelludo.

Verbena teucriifolia Mart. \& Gal. (15). PARTE UTILIZADA: toda la planta. USO: medicinal (padecimientos del oído). FORMA DE EMPLEO: con el cocimiento de la parte aérea se aplican cataplasmas en la zona afectada. 